\title{
Origin of low thermal hysteresis in shape memory alloy ultrathin films
}

\author{
Hongxiang Zong ${ }^{1,2}$, Ze $\mathrm{Ni}^{1}$, Xiangdong Ding ${ }^{1 *}$, Turab Lookman ${ }^{2 *}$, \\ Jun Sun $^{1}$
}

${ }^{1}$ State Key Laboratory for Mechanical Behavior of Materials, Xi'an

Jiaotong University, Xi'an 710049, China

${ }^{2}$ Theoretical Division, Los Alamos National Laboratory, Los Alamos, NM

87545, USA

*Corresponding author:

Xiangdong Ding,Dingxd@mail.xjtu.edu.cn, (86)13379200208

Turab Lookman, txl@lanl.gov, (01)505-665-0419 


\begin{abstract}
Hysteresis in martensitic transformations (MT) limits the usefulness of shape memory alloys (SMAs) in nanosized devices that require high sensitivity, high durability and high energy efficiency. Previous studies have shown that the MT is hindered in the surface region of nanosized SMAs, and therefore there is little hysteresis. However, we find that the hysteretic behavior in SMA nanofilms is not related to the MT suppression. Rather, the decrease in hysteresis is due to weaker spontaneous lattice distortion and spatial heterogeneity, leading to a more continuous phase transformation process. We demonstrate this by designing two classes of nano-sized SMAs, a free-standing Ni62.5Al 37.5 film in which the surface region promotes $\mathrm{MT}$, and a multilayer of $\mathrm{Fe}-\mathrm{Ni}_{62.5} \mathrm{Al}_{37.5-\mathrm{Fe}}$ in which the interface region suppresses MT. Both cases show a decrease in hysteresis with decreasing film thickness. Our findings suggest a method to reduce hysteresis in conventional bulk SMAs.
\end{abstract}

Keywords: size effect; martensitic transformation; shape memory alloys; hysteresis 


\section{Introduction}

A martensitic transformation (MT) enables shape memory alloys (SMAs) to exhibit fascinating properties such as shape memory effect and superelasticity. Based on their unique properties, SMAs have been widely used in sensors, actuators, medical devices, and other applications [1-3]. However, special attention needs to be given to the phenomena of complex hysteresis associated with lack of reversibility under loading due to the application of strain/stress control. This can lead to a reduction in the performance of devices [4-7]. This hysteretic phenomenon is also encountered in many other smart material actuators that involve magnetic materials or piezoelectric materials [8-10]. Therefore, in order to improve the position control accuracy of an SMA actuator, it is necessary to reduce the effects of hysteresis.

It has been shown that reducing the size of SMAs down to the nanoscale (such as SMA nanoparticles, SMA nanocrystallines) can decrease significantly the width of the martenstic transformation hysteresis loop [11-17]. Many theoretical models of martenstic transformations have been developed to explain the small hysteresis in nanosized SMAs, and the one that has performed well is the core-shell model [18-20]. For example, Zhang et al. studied freestanding SMA nanoparticles and showed that the surface region suppressing the B2-B19' transformation locally plays a dominant role in the size dependent transformation behavior, leading to nonhysteretic superelasticity [12, 19]. Sun et al. subsequently used this model to explain the small hysteresis in TiNi nanocrystals [21-22]. Nevertheless, experimental results in other phase transforming systems, such as ferroelectric nanofilms, have shown that the surface region can suppress the spontaneous transformation in a number of cases but can promote phase 
transformation in others [23-25]. Thus, the open questions are (1) what is the hysteresis behavior in the case where the effects of the surface promotes phase transformation? (2) Is the reduction in transformation hysteresis related to the phase transformation occuring in the near-surface region? These two questions and their relationship are still not clear.

In this work, we study the thermal induced phase transformations in nanosized SMAs, aimed at achieving an atomic-level understanding of the hysteresis behavior. The basic idea is as follows: the transformation temperature can be influenced by the surface/interface effect, and we can design two types of ultrathin SMA films (i.e., one with increased transformation temperatures in thin films, the other with decreased transformation temperature) by engineering the surface/interface energies, and then comparing the hysteretic behavior in the two cases. We first use molecular dynamics (MD) simulations to find the relationship between the surface/interface energy and the size dependent characteristic temperatures of the MT. The thermal hysteresis during the martensitic transformation in both cases is then calculated to investigate its relationship to the surface/interface effect. Finally, we discuss the main contributions to the hysteresis behavior in nanosized SMAs.

\section{Methodology}

The ultrathin SMA film model under investigation is created in a model SMA (e.g. $\mathrm{Ni}_{62.5} \mathrm{Al}_{37.5}$ monolayer or model SMA/non-transformed metal (e.g. Ni62.5 $\mathrm{Al}_{37.5} / \mathrm{Fe}$ ) multilayer. The samples are oriented by stacking $\{110\} \mathrm{NiAl}$ atomic planes along the $\mathrm{y}$ axis, with the interfaces located parallel to the x-z plane, as shown in the inset of Fig. 1(a). Periodic boundary conditions are applied in the $\mathrm{x}$ and $\mathrm{z}$ directions while the thickness direction is bounded by two free surfaces. The periodic $\mathrm{x}$ and $\mathrm{z}$ dimensions are $23.98 \mathrm{~nm}$ 
and $33.75 \mathrm{~nm}$, respectively. In order to study the influence of SMA layer thickness on the phase transformations several different thicknesses from $3 \mathrm{~nm}$ to $20 \mathrm{~nm}$ were selected. We adopted an embedded atom method (EAM) interatomic potential developed by Dudarev and Derlet for Fe [26]. A semi-empirical interatomic potential of the form proposed in Ref [27] by Farkas et al. is used to describe the B2-orthorhombic phase transformation in $\mathrm{Ni}_{62.5} \mathrm{Al}_{37.5}$. The interaction potentials of $\mathrm{Fe}$ and $\mathrm{Al} / \mathrm{Ni}$ were based on the usual form used for other metal pairs [28]. These potentials have produced good results for phase transition and defect formation energies [19, 27, 29], however, one should not expect that such semiempirical potentials can reproduce the detailed properties of a specific SMA or material system.

The initial samples were relaxed by quenching with the conjugate gradient algorithm. The samples were then annealed at $1000 \mathrm{~K}$, above the NiAl parent phase stabilizing temperature, for at least 120 picoseconds by using a Nose-Hoover thermostat [30] and Parrinello-Rahman barostat [31] within the isothermal-isobaric ensemble. After this procedure, we performed MD simulations of cooling and heating on the annealed samples utilizing the LAMMPS code [32]. The cooling and heating processes involve cyclic increase or decrease in temperature with rates of $0.01 \mathrm{~K} / \mathrm{ps}$.

\section{Results and Discussion}

\subsection{Thermodynamic model of martenstic transformation in nanosized SMAs}

It is well known that the melting temperature, $\mathrm{T}_{\mathrm{m}}$ in low-dimensional materials can be changed by treating the interface [33-35]. For example, the phenomenon of premelting is commonly observed in free-standing nanometer-sized metal particles, while 
superheating can be obtained when coated by (or embedded in) a high- $\mathrm{T}_{\mathrm{m}}$ materials. The main reason for the different melting behavior is the interface energy $[34,36]$. For nanosized SMAs, a similar strategy can be used to change structural phase transformations. Here $\Delta \gamma$ is defined as the interface energy difference between product phase and parent phase, i.e. $\Delta \gamma=\gamma_{M}-\gamma_{P}$. If a transformation process can reduce the interface energy $(\Delta \gamma<0)$, MT nucleation in the near-interface region will be energetically preferred compared to its bulk counterpart. By contrast, $\Delta \gamma>0$ indicates a suppressing effect on the MT nucleation in this region. Actually, this idea has been used to explain an inverse martensitic transformation in $\mathrm{Zr}$ nanowires [37].

We then derive the relation between transformation temperature and sample size thermodynamically. In analogy with surface melting, we identify the MT by equating the Gibbs free energy $\mu_{P}$ and $\mu_{M}$ of the parent and product phase in nanosized SMA, and the free energy is assumed to be a function of temperature $T_{0}$ and pressure $p_{0}$ :

$\mu_{P}\left(p_{0}, T_{0}\right)=\mu_{M}\left(p_{0}, T_{0}\right)$

This equation means that the free energies of a fully parent phase and martensite are equal at the transition temperature. The free energy can be expanded around its value at the critical point (i.e., transition temperature $T_{0}^{b u l k}$ or transition pressure $p_{0}^{b u l k}$ ), and we retain first-order terms only:

$$
\mu\left(p_{0}, T_{0}\right)=\mu^{\text {bulk }}\left(p_{0}^{\text {bulk }}, T_{0}^{\text {bulk }}\right)+\frac{\partial \mu}{\partial T}\left(T_{0}-T_{0}^{\text {bulk }}\right)+\frac{\partial \mu}{\partial p}\left(p_{0}-p_{0}^{\text {bulk }}\right)
$$

From the Gibbs-Duhem equation $(-V d p+S d T+N d \mu=0)$ it follows that 
$\frac{\partial \mu}{\partial T}=-s, \frac{\partial \mu}{\partial p}=-\frac{1}{\rho}$

where $s=S / n$ is the entropy per atomic and $\rho=n / V$ is the number density. Combined with Equation (1)-(3), and taking into account that $\mu_{p}^{\text {bulk }}\left(p_{0}^{\text {bulk }}, T_{0}^{\text {bulk }}\right)=\mu_{M}^{\text {bulk }}\left(p_{0}^{\text {bulk }}, T_{0}^{\text {bulk }}\right)$, we obtain:

$-S_{P}\left(T_{0}-T_{0}^{\text {bulk }}\right)+\frac{1}{\rho_{P}}\left(p_{p}-p_{0}^{\text {bulk }}\right)=-S_{M}\left(T_{0}-T_{0}^{\text {bulk }}\right)+\frac{1}{\rho_{M}}\left(p_{M}-p_{0}^{\text {bulk }}\right)$

Here one must distinguish between the pressure $p_{p}$ of the parent phase and the pressure $p_{M}$ of a martensite because of the Laplace contribution $p=2 \gamma / r$, where $\gamma$ is the interface energy or interface tension and $r$ is its radius. Since parent phase and product should have different interface energy $\gamma$ in nanosized SMAs $\left(\gamma_{P}\right.$ for parent phase, $\gamma_{M}$ for product phase ), we obtain:

$$
-S_{P}\left(T_{0}-T_{0}^{b u l k}\right)+\frac{2 \gamma_{P}}{\rho_{P} r_{P}}=-S_{M}\left(T_{0}-T_{0}^{b u l k}\right)+\frac{2 \gamma_{M}}{\rho_{M} r_{M}}
$$

For the solid to solid transition, the volume change is insignificant, and can be neglected, that is $\rho_{P}=\rho_{M}=\rho, r_{p}=r_{M}=D / 2, D$ is the nanoparticle diameter or film thickness. Taking into account that $\Delta H=T_{0}^{b u l k}\left(s_{P}-s_{M}\right)$ is the latent heat per atom during the phase transformation, the relation between $T_{0}$ and $D$ is then obtained as

$$
T_{0}=T_{0}^{b u l k}-\left(2 T_{0}^{b u l k} \rho_{0}^{-1} \Delta H_{0}^{-1}\right) \frac{\Delta \gamma}{D}
$$

where $T_{0}^{\text {bulk }}$ and $2 \rho_{0}^{-1} \Delta H_{0}^{-1}$ are constant. 


\subsection{Size effects on phase transformation in nanosized SMAs}

For miniaturized applications such as actuators, magnetic cooling, and hybrid systems, thin films are often mandatory. Here, we simulated the martenstic transformation behavior in nanosized SMA films. Our results show that the size effect on MT temperatures of freestanding SMA monolayers and SMA multilayers is quite different. The transformation temperature $\mathrm{T}_{0}$ increases with the film thickness, while $\mathrm{T}_{0}$ is reduced in thinner SMA multilayer.

\subsubsection{Evolution of Martensitic transformation in SMA thin films}

Fig. 1(a) shows the temperature dependent potential energy of the freestanding SMA monolayer (with the thickness $\mathrm{D}=10 \mathrm{~nm}$ ) during the cooling and heating process. Under cooling the potential energy decreases smoothly with temperature. Then a discontinuous drop occurs near $\mathrm{M}_{\mathrm{s}}=475 \mathrm{~K}$, indicating the occurrence of a first-order phase transformation. For the martensitic transformation (MT) in $\mathrm{Ni}_{62.5} \mathrm{Al}_{37.5}$, the highsymmetry cubic phase undergoes a transformation to orthorhombic martensite. The corresponding microstructure evolution is demonstrated in the left panels of Fig. 2. The $\mathrm{Ni}_{62.5} \mathrm{Al}_{37.5}$ atoms are colored according to their local atomic packing determined from characteristic bond angle analysis [38]. Atoms with a cubic environment are shown as light blue spheres and represent austenite, orange spheres denote a local orthorhombic environment and mark the martensite phase, other atoms belong to defects (such as free surface, domain boundaries). By examining multiple snapshots stored during the MD simulations, we find that the MT process is mediated by a metastable state or distorted parent phase (green atoms in Fig. 2). The MT begins with the formation of the metastable 
phase from the near-surface region of SMA films, and martensite then nucleates within the distorted parent phase region (Fig. 2(b) and 2(c)). A further cooling leads to the entirely orthorhombic phase with multi-domain structure, as shown in Fig. 1(d). The multidomain structure consists of lath-like domains and domain walls paralleled to the free surface, which is different from their bulk counterpart. It is interesting to note that the domain walls are mobile in the low-temperature phase (Fig. 2(d) and 2(e)).

Upon heating, the reverse martensitic transformation takes place at a much higher temperature $A_{s}=1120 \mathrm{~K}$ compared to the forward phase transformation (Fig. 1(a)), indicating significant thermal hysteresis (close to $800 \mathrm{~K}$ ). The associated microstructure is shown in the right panels of Fig. 2. Unlike the forward MT, the parent phase prefers to nucleate from both domain walls and free surfaces (Fig. 2(f)), and then grows along the thickness direction (Fig. 2(g)), transforming back to a parent phase identical to the original microstructure when it is heated above $A_{f}$ (Fig. 2(h)). Thus, a phase transformation cycle in ultrathin SMA films, similar to that of bulk materials, is reproduced by the present $\mathrm{Ni}_{62.5} \mathrm{Al}_{37.5}$ monolayer model.

\subsubsection{Size effect on martensitic transformation in freestanding SMA thin films}

Most studies of phase transformations within SMA films involve substratesupported films and the substrate leads to significant mechanical. However, little is known about the freestanding SMA films. For structural phase transformations in freestanding thin films, surface conditions (such as surface energy or surface reconstruction) should be a key factor. Therefore, we explore how film thickness affects the transformation temperature and martensitic microstructure within freestanding SMA 
films.

Fig. 1(b) shows the forward and reverse transformation temperatures (Ms and $A_{f}$, respectively) of six $\mathrm{Ni}_{62.5} \mathrm{Al}_{37.5}$ freestanding films with a (110) free surface, ranging from 3.0 to $20.0 \mathrm{~nm}$ in thickness. As the film thickness decreases, Ms increases and $\mathrm{A}_{\mathrm{f}}$ is essentially constant. Subsequently, we calculated the corresponding transformation temperatures $T_{0}$ as a function of film thickness, where $T_{0}=\left(M s+A_{f}\right) / 2$, and the result is shown in Fig. 3(a). We noticed that the freestanding SMA films with (110) free surface show an inverse size effect, i.e., $\mathrm{T}_{0}$ increase with the decrease of film thickness, differing from the previous experimental observations and MD simulations based on SMA nanparticles $[17,39-40]$. We found that this inverse size effect can be understood by our analytical model (Equation (6)) with the scenario of $\Delta \gamma<0$. This indicates that the surface effect promotes phase transformation in freestanding $\mathrm{Ni}_{62.5} \mathrm{Al}_{37.5}$ films. It is consistent with the corresponding microstructure evolution shown in Fig. 2, where the MT nucleates from the near-surface region.

We then calculate the thermal hysteresis during the forward and reverse MT in (110) freestanding films. The film thickness dependent phase transformation hysteresis $\Delta \mathrm{T}$ (here we define $\Delta \mathrm{T}=\mathrm{As}-\mathrm{Ms}$ ) is also shown in Fig. 3(b). The MD simulations show that the thermal hysteresis $\Delta \mathrm{T}$ decreases with the reduction of film thickness. This means that the SMA nano-films with surface promoting effect of MT show a hysteresis reduction when the feature size decreases. Interestingly, the well-observed experimental and MD results on nano-particles $[12,19]$, where the near surface region strongly suppressing MT, also show the reduction of hysteresis with the particle size. All these indicate that the size dependent hysteresis behavior may not strongly correlate with phase transformation in 
the near-surface region.

In order to check the reliability of the above results, we simulated freestanding films with two other orientations, i.e. (111) and (112) free surfaces, as shown in Fig. 3. As expected, similar promoting transformation temperatures and reduced thermal hysteresis are obtained in all cases, although (110) film has higher transformation temperatures and lower hysteresis than the other two orientations due to the difference of surface energy.

In addition, we take (110) film as an example, investigate the changes of corresponding microstructures due to the size effect, as shown in Fig. 4. The atomic configurations obtained from our MD simulations are shown in the left panel of Fig. 4, and the right panel indicates the corresponding order parameter orientations of the martensite variants. A size effect in domain structures is observed: a transition from predominantly multidomais to single-domains occurs with decreasing film thickness. Specifically, the thinnest $\mathrm{Ni}_{62.5} \mathrm{Al}_{37.5}$ film $(2.5 \mathrm{~nm})$ in Fig. 4(a) exhibits a single domain structure; however, when the film thickness increases to $5.0 \mathrm{~nm}$, a two-dimensional multidomain structure with in-plane domain walls (DWs) is obtained (Fig. 4(b)). The thickness increases up to $14.8 \mathrm{~nm}$ and the microstructure of the low-temperature phase grows into out-of-plane multidomain structures, the DWs of which are distributed more randomly, as shown in Fig. 4(c). The development of DWs in martensite is often considered as a driving force for the reduction in internal stress. A three-dimensional multidomain structure is able to release the internal stresses and consequently lower their free energies in bulk materials or considerable thick films $(>14.8 \mathrm{~nm})$ in our case. With reducing film thickness, the tendency of the domain to rotate out-of-plane, however, is hindered by the presence of free surfaces. As a result, the internal elastic strain energy (or 
the shape anisotropy) is smallest when the order parameter lies in the plane of the film. Eventually, single-domain films become stable and dominating, i.e., the formation of new domains (nucleation) or domain walls is not allowed when the film thickness is smaller than $2.5 \mathrm{~nm}$.

\subsubsection{Size effect on martensitic transformation in multilayered SMA films}

Figure 5 shows size effects associated with martensitic transformation in multilayered SMA films, i.e., $\mathrm{Ni}_{62.5} \mathrm{Al}_{37.5}$ films on alternating Fe layers (the inset of Fig. 5(a)). The orientation relationship corresponds to $\{110\}_{\mathrm{Fe}}\left\|\{110\}_{\mathrm{NiAl}}\right\|$ interface plane and $\langle 110\rangle_{\mathrm{Fe}} \|$ $<001>_{\mathrm{NiAl}}$ in the interface plane. Compared to the freestanding monolayer films, the MT of $\mathrm{NiAl}$ in multilayered $\mathrm{Fe} / \mathrm{Ni}_{62.5} \mathrm{Al}_{37.5} / \mathrm{Fe}$ films is suppressed, i.e., the transformation temperatures $\mathrm{T}_{0}$ decreased when the thickness of NiAl film $\mathrm{D}$ is reduced, as shown in Fig. 5(a). We find that $\mathrm{T}_{0}$ first decreases slowly with decreasing film thickness when $\mathrm{D}>6 \mathrm{~nm}$. Below the critical size $D=6 \mathrm{~nm}, \mathrm{~T}_{0}$ is more sensitive to the film thickness, which is similar to the size effect observed in freestanding SMA nanoparticles [19]. This case should agree with the scenario of $\Delta \gamma>0$ in our analytical model (Equation (6)). Subsequently, we checked the MT process by examining the corresponding snapshots during cooling. As shown in Fig. 6, the martensite nucleates from the inner side of the NiAl layer instead of the near-surface region (Fig. 6(a)), and it then grows along the thickness direction, forming an entirely martensite with complex multi-domain structure (Fig. 6(b), 6(c) and 6(d)). This indicates that the surface effect suppresses phase transformation in multilayered SMA films.

We also calculated thermal hysteresis $\Delta \mathrm{T}$ as a function of the NiAl film thickness, as 
shown in Fig. 5(b). We notice that $\Delta \mathrm{T}$ decrease with the film thickness in multilayered SMA films, indicating a similar hysteresis behavior to MT in nanoparticles [40] or nanocrystallites [16-17]. Thus, we can summarize that hysteresis behavior does not strongly correlate with phase transformation in the near-surface region in nanosized SMAs.

\subsection{Comparison of analytical model with simulation results}

In order to compare our MD simulation results with the previous thermodynamic model, we calculate the surface/interface energy difference $\Delta \gamma . \Delta \gamma$ in our MD simulations is defined as follows:

$$
\Delta \gamma=\left(E_{M}^{\text {nano }}-E_{M}^{\text {Bulk }}\right)-\left(E_{P}^{\text {nano }}-E_{P}^{\text {Bulk }}\right)
$$

where $E_{i}^{\text {nano }}$ is the total potential energy of the $i$ phase in nanosized SMA samples, $E_{i}^{\text {Bulk }}$ are the corresponding potential energy of bulk materials. In order to decrease the relative errors in $\mathrm{T}_{0}$ due to the random substitution of $\mathrm{Ni}$ atoms in the $\mathrm{NiAl}$ alloy, we calculate each $\Delta \gamma$ three times. Fig. 7(a) shows that $\Delta \gamma$ as a function of film thickness or particle diameter, $\Delta \gamma$ in nanosized SMAs also has a weak size-dependent. With decreasing sample size, both the freestanding SMA samples and the multilayered samples show a slightly decreasing or increasing in surface/interface energy difference, and the multilayered films show a stronger size-dependence due to the mechanical constraint by the Fe substrate. It is important to note that the sign of $\Delta \gamma$ is consistent with our previous speculation. i.e., $\Delta \gamma<0$ for all freestanding SMA films, while $\Delta \gamma>0$ for the multilayered SMA films and SMA nanoparticles.

In addition, we find that the simulation data for both cases can be linear fitted well with the $\mathrm{T}_{0} \sim-\Delta \gamma / \mathrm{D}$ relation in Equation (6), as shown in Fig. 7(b). Our simulation 
results are also consistent with previous studies on structural phase transformation in $\mathrm{Ni}$ Al nanoparticles, Fe-Ni nanoparticles and Fe nanowires, i.e., the $\mathrm{T}_{0} \sim \mathrm{D}^{-1}$ relation [4143]. However, precious models on size effects associated with martensitic transformation mainly are based on the phenomenological parameter $\delta$ (extrapolation length). The present study indicates that the surface energy difference during the MT is the dominant factor at the atomic level.

\subsection{Discussion of low thermal hysteresis in nanosized SMAs}

Although the size effect on MT temperatures of freestanding SMA monolayers and SMA multilayers is quite different, a similar hysteretic behavior of SMA is obtained in both cases, i.e., the transformation hysteresis decrease with SMA size. Therefore, we cannot simply contribute the reduction of hysteresis to the MT suppression in the nearsurface region. Our discussions below indicate that low thermal hysteresis in nanosized SMAs actually stems from the reduced spontaneous lattice distortion and spatial heterogeneity of order parameter.

\subsubsection{Weakening of spontaneous lattice distortion}

For SMA bulk, the thermal hysteresis arises largely from the generation and motion of internal interfaces (i.e., phase boundaries and twin boundaries) during the MT process [42-44]. Since the surface breaks the translational invariance of the system, a different behavior on the microstructural evolution is expected. Thus, we first use order parameters to explain the evolution of martensitic transformation within the nanosized SMAs phenomenologically. For the B2-orthorhombic $\mathrm{MT}$ in $\mathrm{Ni}_{62.5} \mathrm{Al}_{37.5}$, the order parameters are the orthorhombic lattice distortions, which can be estimated by following equation: 
$\eta \approx \frac{\cos \theta}{\cos \theta_{0}}-1$

where $\theta\left(<\theta_{0}\right)$ is the angle between two body diagonals of the unit cell shown in the inset of Fig. 8(b), $\theta_{0}$ is the body diagonal angle for the perfect parent phase. We then calculate the spatial distribution of the average $\eta$ along the thickness direction during the cooling process.

The evolution of $\eta$ in nanosized SMA samples during the phase transformation is shown in Fig. 8. Fig. 8(a) and 8(b) compare two freestanding films with different film thickness D. In both cases, $\eta$ in the surface region increases firstly and then grows to the middle in both samples, consistent with atomic-level images in Fig. 2. However, in contrast to the $10 \mathrm{~nm}$-thick SMA films (see Fig. 8(a)), the spontaneous lattice distortion in $\mathrm{D}=5 \mathrm{~nm}$ films is much reduced (Fig. 8(b)). This reduction is also confirmed in the multilayered SMA films, as shown in Fig. 8(c) and 8(d).

The Landau theory explains the first-order nature of MT and the hysteretic behavior of bulk SMAs [45]. Unlike a second-order phase transition, there still exists an energy barrier between parent phase and martensite when the temperature approaches $\mathrm{T}_{0}$. The energy barrier leads to the presence of multiple metastable two-phase configurations, and the dynamics connecting these configurations dissipates a significant fraction of input energy. However, the reduction of spontaneous lattice distortion in nanosized SMA films will change the MT from strongly first-order to weakly first-order transition (see Fig. 9(a)), and the energy barrier between the parent phase and martensite will also be reduced. From this point of view, the lower limit should be a continuous phase transition (almost a second-order), where the energy barrier for the phase transition vanishes, and gives rise 
to zero-hysteresis.

\subsubsection{Spatial heterogeneity}

As shown in Fig. 8, the spontaneous lattice distortion in nanosized SMA films is not spatially uniform. $\eta$ in the near-surface region is quite different from the interior, and the spatial heterogeneity of $\eta$ is also enhanced in thinner SMA films. It's important to note that this heterogeneity also plays an important role in the low MT hysteresis. Specifically, the spatial heterogeneity can change the simple two-wall free energy landscape into a complex one with multiple energy minima. In addition, the resulting metastable states along the transformation pathway give rise to a decrease in the energy barrier, thus leading to the reduction of thermal hysteresis, as shown in Fig. 9(b). The same mechanism has been found in strain glass alloys, where point defects induced inhomogeneity gives rise to superelasicity with slim hysteresis [46-47]. In addition, the low gradient of $\eta$ across the phase boundary in nanosized SMAs indicates a more continuous MT process, which can result in reduced energy dissipation during phase boundary motion (Fig. 8).

In order to confirm the spatial heterogeneity in free energy $\mathrm{F}=U-T_{0} \Delta S$ at $\mathrm{T}_{0}$, we then calculated the spatial distribution of internal energy $U$ and entropy $\Delta S$ inside SMA films, as shown in Fig. 10. Here we estimate entropy by employing the Debye model for the vibrational spectrum, which provides a simple relation between entropy and mean square displacement (MSD) [48]. By parametrizing an effective Debye model for the vibrational spectra of the two phases of interest, we can approximate their entropy difference by:

$$
\Delta S=0.5 k_{B} \ln \left(\left\langle\Delta r_{2}^{2}\right\rangle /\left\langle\Delta r_{1}^{2}\right\rangle\right)
$$


where $\left\langle\Delta r_{i}^{2}\right\rangle$ is the MSD of $i$ phase. We choose four different freestanding SMA films with a film thickness range from $2.5 \mathrm{~nm}$ to $10 \mathrm{~nm}$, and the relaxation temperature keeps $\mathrm{T}_{0}=\left(\mathrm{Ms}+\mathrm{A}_{\mathrm{f}}\right) / 2$.

Figures 10(a) and 10(b) show the average atomic potential energies, and Figs. 10(c) and 10(d) depict the corresponding MSD as a function of distance to the centers of the freestanding films. The potential energy and MSD profiles have similar shapes with a constant region in the core that remains unchanged as the film thickness increases. Both potential energies and MSD increase steeply in the near-surface layer, which is consistent with the core-shell model used in other nano-materials $[12,48]$. As compared to the SMA bulk behavior, the inhomogeneity of the internal energy and entropy induced by the surface regions can smear the first-order transformation and lower the martensitic transformation hysteresis. As shown in Fig. 10, the volume fraction of atoms located in the surface region increases with the reduced film thickness, giving rise to a decreasing thermal hysteresis.

\section{Conclusion}

We have used $\mathrm{Ni}_{62.5} \mathrm{Al}_{37.5}$ films as a model SMA system to study the hysteresis behavior associated with martenstic transformation in nanosized ultrathin films. Our findings can be summarized as following: (1) Size dependent transformation temperatures in ultrathin SMA films can be changed by surface/interface energy engineering; (2) The thermal hysteresis of MT decreases with sample size in nanosized SMAs, and is not related to MT suppression in the surface region. (3) The reduced MT hysteresis in nano-sized SMAs is dominated by weak spontaneous strain and spatial 
heterogeneity, giving rise to a quasi-continuous phase transformation process. Due to the structural transformation in wide ferroic film materials, our findings potentially have board applications in functional nanosystems such as shape memory effect, ferroelectric and magnetocaloric systems.

\section{Acknowledgments}

This work was supported by NSFC (51171140, 51231008, 51320105014 and 51321003), the 973 Program of China (2012CB619402) and 111 project (B06025), as well as US DOE at LANL (DE-AC52-06NA25396). 


\section{References:}

[1] M.A. McEvoy, N. Correll. Materials that couple sensing, actuation, computation, and communication, Science 347 (2015) 6228.

[2] J. Mohd Jani, M. Leary, A. Subic, M.A. Gibson. A review of shape memory alloy research, applications and opportunities, Mater. Design 56 (2014) 1078-1113.

[3] K. Otsuka, T. Kakeshita. Science and technology of shape-memory alloys. New developments, MRS Bull. 27 (2002) 91-100.

[4] K. Otsuka, X. Ren. Physical metallurgy of Ti-Ni-based shape memory alloys, Prog. Mater. Sci. 50 (2005) 511-678.

[5] C. Chluba, W. Ge, R. Lima de Miranda, J. Strobel, L. Kienle, E. Quandt, M. Wuttig. Ultralow-fatigue shape memory alloy films, Science 348 (2015) 1004-1007.

[6] K.K. Ahn, N.B. Kha. Improvement of the performance of hysteresis compensation in SMA actuators by using inverse Preisach model in closed-loop control system, J. Mech. Sci. Tech. 20 (2006) 634-642.

[7] R. Kainuma, Y. Imano, W. Ito, Y. Sutou, H. Morito, S. Okamoto, O. Kitakami, K. Oikawa, A. Fujita, T. Kanomata, K. Ishida. Magnetic-field-induced shape recovery by reverse phase transformation, Nature 439 (2006) 957-960.

[8] A. Sozinov, A.A. Likhachev, N. Lanska, K. Ullakko. Giant magnetic-field-induced strain in NiMnGa seven-layered martensitic phase, Appl. Phys. Lett. 80 (2002) 17461748 .

[9] O. Auciello, J.F. Scott, R. Ramesh. The physics of ferroelectric memories, Phys. Today 51 (1998) 22-27.

[10]C.A.P. Dearaujo, J.D. Cuchiaro, L.D. McMillan, M.C. Scott, J.F. Scott. Fatigue-free ferroelectric capacitors with platinum-electrodes, Nature 374 (1995) 627-629.

[11]R. Mirzaeifar, K. Gall, T. Zhu, A. Yavari, R. DesRoches. Structural transformations in NiTi shape memory alloy nanowires, J. Appl. Phys. 115 (2014) 194307.

[12]Z. Zhen, D. Xiangdong, S. Jun, T. Suzuki, T. Lookman, K. Otsuka, R. Xiaobing. Nonhysteretic superelasticity of shape memory alloys at the nanoscale, Phys. Rev. Lett. 111 (2013) 145701.

[13]A. Ahadi, Q. Sun. Effects of grain size on the rate-dependent thermomechanical responses of nanostructured superelastic NiTi, Acta Mater. 76 (2014) 186-197. 
[14]N. Teichert, A. Auge, E. Yuzuak, I. Dincer, Y. Elerman, B. Krumme, H. Wende, O. Yildirim, K. Potzger, A. Huetten. Influence of film thickness and composition on the martensitic transformation in epitaxial Ni-Mn-Sn thin films, Acta Mater. 86 (2015) 279-285.

[15]K. R. Morrison, M. J. Cherukara, Hojin Kim, A. Strachan. Role of grain size on the martensitic transformation and ultra-fast superelasticity in shape memory alloys, Acta Mater. 95 (2015), 37-43.

[16]R. Ahluwalia, S. S. Quek, David T. Wu. Simulation of grain size effects in nanocrystalline shape memory alloys, J. Appl. Phys. 117 (2015) 244305.

[17] Y. Sutou, T. Omori, K. Yamauchi, N. Ono, R. Kainuma, K. Ishida. Effect of grain size and texture on pseudoelasticity in $\mathrm{Cu}-\mathrm{Al}-\mathrm{Mn}$-based shape memory wire, Acta Mater. 53 (2005) 4121-4133.

[18]Z. Wang, J. Hu, M.-F. Yu. Axial polarization switching in ferroelectric BaTiO3 nanowire, Nanotechnology 18 (2007) 235203.

[19]Z. Zhang, X. Ding, J. Deng, J. Cui, J. Sun, T. Suzuki, K. Otsuka, X. Ren. Surface Effects on structural phase transformations in nanosized shape Memory Alloys, J. Phys. Chem. C 117 (2013) 7895-7901.

[20] J.M. Kiat, C. Bogicevic, P. Gemeiner, A. Al-Zein, F. Karolak, N. Guiblin, F. Porcher, B. Hehlen, L. Yedra, S. Estradé, F. Peiró, R. Haumont. Structural investigation of strontium titanate nanoparticles and the core-shell model, Phys. Rev. B 87 (2013) 024106.

[21]Q. Sun, A. Aslan, M. Li, M. Chen. Effects of grain size on phase transition behavior of nanocrystalline shape memory alloys, Sci. China Technol. Sci. 57 (2014) 671-679.

[22]A. Ahadi, Q. Sun. Stress-induced nanoscale phase transition in superelastic NiTi by in situ X-ray diffraction, Acta Mater. 90 (2015) 272-281.

[23]J. Hong, D. Fang. Size-dependent ferroelectric behaviors of BaTiO3 nanowires, Appl. Phys. Lett. 92 (2008) 012906.

[24] J.F. Scott, H.M. Duiker, P.D. Beale, B. Pouligny, K. Dimmler, M. Parris, D. Butler, S. Eaton. Properties of ceramic KNO3 thin-film memories, Physica B \& C 150 (1988) 160-167.

[25]C. Upadhyay, P.K. Harijan, A. Senyshyn, R. Ranganathan, D. Pandey. Extraordinary 
enhancement of Néel transition temperature in nanoparticles of multiferroic tetragonal compositions of $(1-\mathrm{x}) \mathrm{BiFeO} 3-\mathrm{xPbTiO} 3$ solid solutions, Appl. Phys. Lett. 106 (2015) 093103.

[26] S.L. Dudarev, P.M. Derlet. A 'magnetic' interatomic potential for molecular dynamics simulations, J. Phys.: Condens. Matter. 17 (2005) 7097.

[27]D. Farkas, B. Mutasa, C. Vailhe, K. Ternes. Interatomic potentials for B2 NiAl and martensitic phases, Model. Simul. Mater. Sci. Eng. 3 (1995) 201.

[28]R.A. Johnson. Alloy models with the embedded-atom method, Phys. Rev. B 39 (1989) 12554-12559.

[29] K.R. Morrison, M.J. Cherukara, K. Guda Vishnu, A. Strachan. Role of atomic variability and mechanical constraints on the martensitic phase transformation of a model disordered shape memory alloy via molecular dynamics, Acta Mater. 69 (2014) 30-36.

[30] G.J. Martyna, M.L. Klein, M. Tuckerman. Nose-Hoover chains - the canonical ensemble via continuous dynamics, J. Chem. Phys. 97 (1992) 2635-2643.

[31]M. Parrinello, A. Rahman. Crystal structure and pair Potentials: a moleculardynamics study, Phys. Rev. Lett. 45 (1980) 1196-1199.

[32] S. Plimpton. Fast parallel algorithms for short-range molecular-dynamics, J. Comput. Phys. 117 (1995) 1-19.

[33]L.J. Lewis, P. Jensen, J.L. Barrat. Melting, freezing, and coalescence of gold nanoclusters, Phys. Rev. B 56 (1997) 2248-2257.

[34]K. Lu, Z.H. Jin. Melting and superheating of low-dimensional materials, Curr. Opin. Solid State Mater. Sci. 5 (2001) 39-44.

[35]Z.L. Wang, J.M. Petroski, T.C. Green, M.A. El-Sayed. Shape transformation and surface melting of cubic and tetrahedral platinum nanocrystals, J. Phys. Chem. B 102 (1998) 6145-6151.

[36]F. Baletto, R. Ferrando. Structural properties of nanoclusters: Energetic, thermodynamic, and kinetic effects, Rev. Mod. Phys. 77 (2005) 371-423.

[37]S. Li, X. Ding, J. Li, X. Ren, J. Sun, E. Ma, T. Lookman. Inverse martensitic transformation in Zr nanowires, Phys. Rev. B 81 (2010) 245433.

[38]G.J. Ackland, A.P. Jones. Applications of local crystal structure measures in 
experiment and simulation, Phys. Rev. B 73 (2006) 054104.

[39] K. Kadau, M. Gruner, P. Entel, M. Kreth. Modeling structural and magnetic phase transitions in iron-nickel nanoparticles, Phase Transit. 76 (2003) 355-365.

[40]D. Mutter, P. Nielaba. Simulation of the thermally induced austenitic phase transition in NiTi nanoparticles, Eur. Phys. J. B 84 (2011) 109-113.

[41]D.D. Fong, G.B. Stephenson, S.K. Streiffer, J.A. Eastman, O. Auciello, P.H. Fuoss, C. Thompson. Ferroelectricity in ultrathin perovskite films, Science 304 (2004) 16501653.

[42]P.J. Shamberger, F.S. Ohuchi. Hysteresis of the martensitic phase transition in magnetocaloric-effect Ni-Mn-Sn alloys, Phys. Rev. B 79 (2009) 144407.

[43]J. Cui, Y.S. Chu, O.O. Famodu, Y. Furuya, J. Hattrick-Simpers, R.D. James, A. Ludwig, S. Thienhaus, M. Wuttig, Z.Y. Zhang, I. Takeuchi. Combinatorial search of thermoelastic shape-memory alloys with extremely small hysteresis width, Nat. Mater. 5 (2006) 286-290.

[44] A. Bekker, L.C. Brinson. Phase diagram based description of the hysteresis behavior of shape memory alloys, Acta Mater. 46 (1998) 3649-3665.

[45]M. Brokate, J. Sprekels. Hysteresis and Phase Transitions, Springer New York, 2012.

[46]D. Wang, S. Hou, Y. Wang, X. Ding, S. Ren, X. Ren, Y. Wang. Superelasticity of slim hysteresis over a wide temperature range by nanodomains of martensite, Acta Mater. 66 (2014) 349-359.

[47]L. Berthier, G. Biroli. Theoretical perspective on the glass transition and amorphous materials, Rev. Mod. Phys. 83 (2011) 587-645.

[48]P. Erhart, B. Sadigh. Low-temperature criticality of martensitic transformations of $\mathrm{Cu}$ nanoprecipitates in $\alpha-F e$, Phys. Rev. Lett. 111 (2013) 025701. 


\section{Figure Captions}

FIG. 1 The film thickness dependent martensitic transformation characteristic temperatures in free-standing (110) $\mathrm{Ni}_{63.5} \mathrm{Al}_{37.5}$ monolayer. (a) Variation of the average potential energy of a $\mathrm{Ni}_{63.5} \mathrm{Al}_{37.5}$ film with $10 \mathrm{~nm}$ in thickness during cooling and heating. The inset shows the crystallographic orientation of the (110) $\mathrm{Ni}_{63.5} \mathrm{Al}_{37.5}$ monolayer simulated. (b) The transformation temperatures, Ms and $\mathrm{A}_{\mathrm{f}}$ (arrows in a), as a function of the film thickness D.

FIG. 2 Typical microstructure evolution of $\mathrm{D}=10 \mathrm{~nm}$ freestanding (110) monolayer during cooling and heating. (a)-(d) The martensite nucleates at the free surface, and then grows towards the interior of the SMA film, forming a domain boundary. (e)-(h) The reverse transformation starts from the domain boundary and the free surface, then grows almost to the parent phase. The light blue color represents the ideal bcc structure, green is the distorted bcc structure, the orange represents the ideal orthorhombic structure, and other colors belong to defects.

FIG. 3 Size effects associated with the martenstic transformation in freestanding SMA films. (a) The film thickness dependent transformation temperatures $T_{0}=\left(M s+A_{f}\right) / 2$ for (111), (112) and (110) $\mathrm{Ni}_{63.5} \mathrm{Al}_{37.5}$ films. (b) The corresponding transformation hysteresis $\Delta \mathrm{T}=\mathrm{As}-\mathrm{Ms}$ as a function of the film thickness $\mathrm{D}$ during the forward and reverse phase transformations. Error bars show the standard deviation of $\mathrm{T}_{0}$ and $\Delta \mathrm{H}$ at each thickness. 
FIG. 4 The film thickness dependent atomic configurations of martensite variants in a freestanding SMA monolayer. (a) A single variant formed in the $2.5 \mathrm{~nm}$ ultrathin films. (b) The product of the $5.0 \mathrm{~nm}$ film consists of a multi-variants structure with in-plane order parameter state. (c) A multi-variants structure with out-of-plane order parameter state is formed in the $14.8 \mathrm{~nm}$ films. DW represents the domain wall, which separates different martensite variants. The dashed rhombic cells indicate the order parameter orientations of the martensite variants, and the green solid lines indicate domain walls.

FIG. 5 Size effects associated with the martenstic transformation in multilayered SMA films and freestanding nanoparticles. (a) The size dependent transformation temperatures $\mathrm{T}_{0}=\left(\mathrm{Ms}+\mathrm{A}_{\mathrm{f}}\right) / 2$ for $\mathrm{Ni}_{63.5} \mathrm{Al}_{37.5}$ multilayers. (b) The corresponding transformation hysteresis $\Delta \mathrm{H}=\mathrm{As}-\mathrm{Ms}$ as a function of the size during the forward and reverse phase transformations. Error bars show the standard deviation of $\mathrm{T}_{0}$ and $\Delta \mathrm{T}$ at each size.

FIG. 6 Typical microstructure evolution of $\mathrm{D}=10 \mathrm{~nm}$ in multilayered SMA films during the cooling process. Here we only show the SMA layer, martensite nucleates from the interior of the SMA film and then grows to form multi-domain. The light blue color represents the ideal bcc structure, green is the distorted bcc structure, the orange represents the ideal orthorhombic structure, and other colors belong to defects.

FIG. 7 Interface energy difference dependent size effects of martensitic transformation. (a) 
Interface energy difference $\Delta \gamma$ during the martensitic transformation for freestanding SMA monolayers and multilayers. The dash line shows the interface energy difference for multilayered SMA films. (b) Phase transformation temperature as a function of $\Delta \gamma / D$, a linear relation fitted by $T_{0}-T_{0}^{\text {bulk }} \propto \Delta \gamma / D$.

Fig. 8 Spatial distribution of spontaneous lattice distortion during phase transformation for SMA nanofilms. We compared the evolution of the order parameter distribution in freestanding films with thicknesses (a) $10 \mathrm{~nm}$ and (b) $5 \mathrm{~nm}$, as well as multilayered films with thicknesses (c) $6.4 \mathrm{~nm}$ and (d) $3.2 \mathrm{~nm}$, showing that the MT is more continuous and weaker in thinner films. The inset to (b) shows the definition of order parameter $\eta, \theta$ is the angle between the two body diagonals.

Fig. 9 Schematic illustration of the influence of size on the temperature dependent (a) order parameters and (b) free energy landscape.

Fig. 10 The spatial heterogeneity of the freestanding SMA ultrathin films. (a) and (b) show spatial distribution of potential energy for parent phase and martensite at $\mathrm{T}_{0}$, respectively. (c) and (d) show mean square displacement of a function of distance from the film center for parent phase and martensite at $\mathrm{T}_{0}$, respectively. 

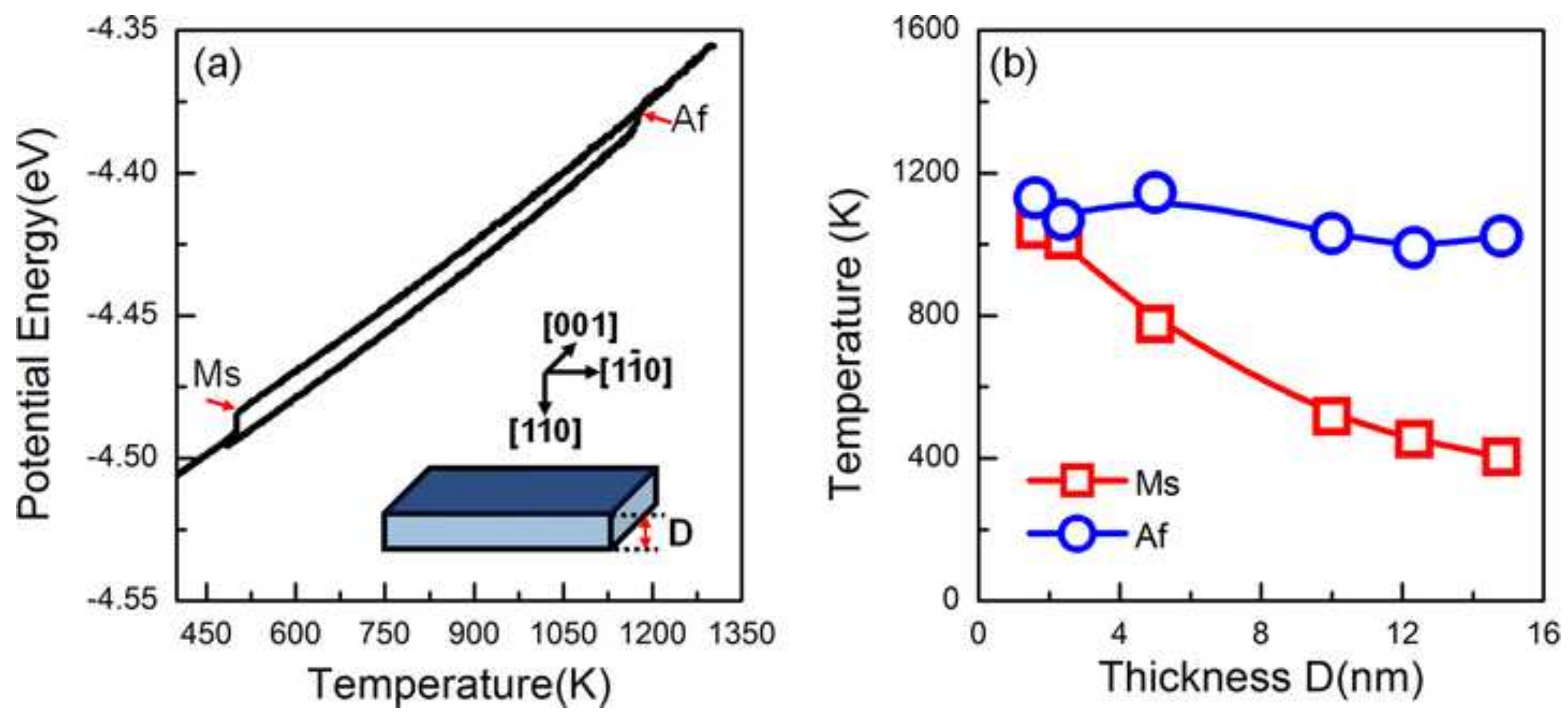
(a)

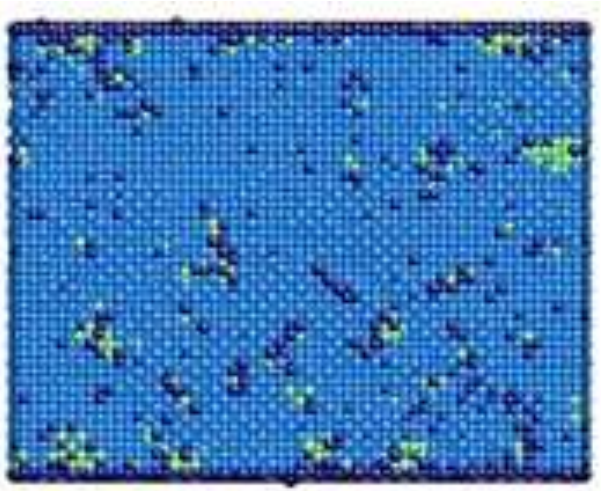

(b)

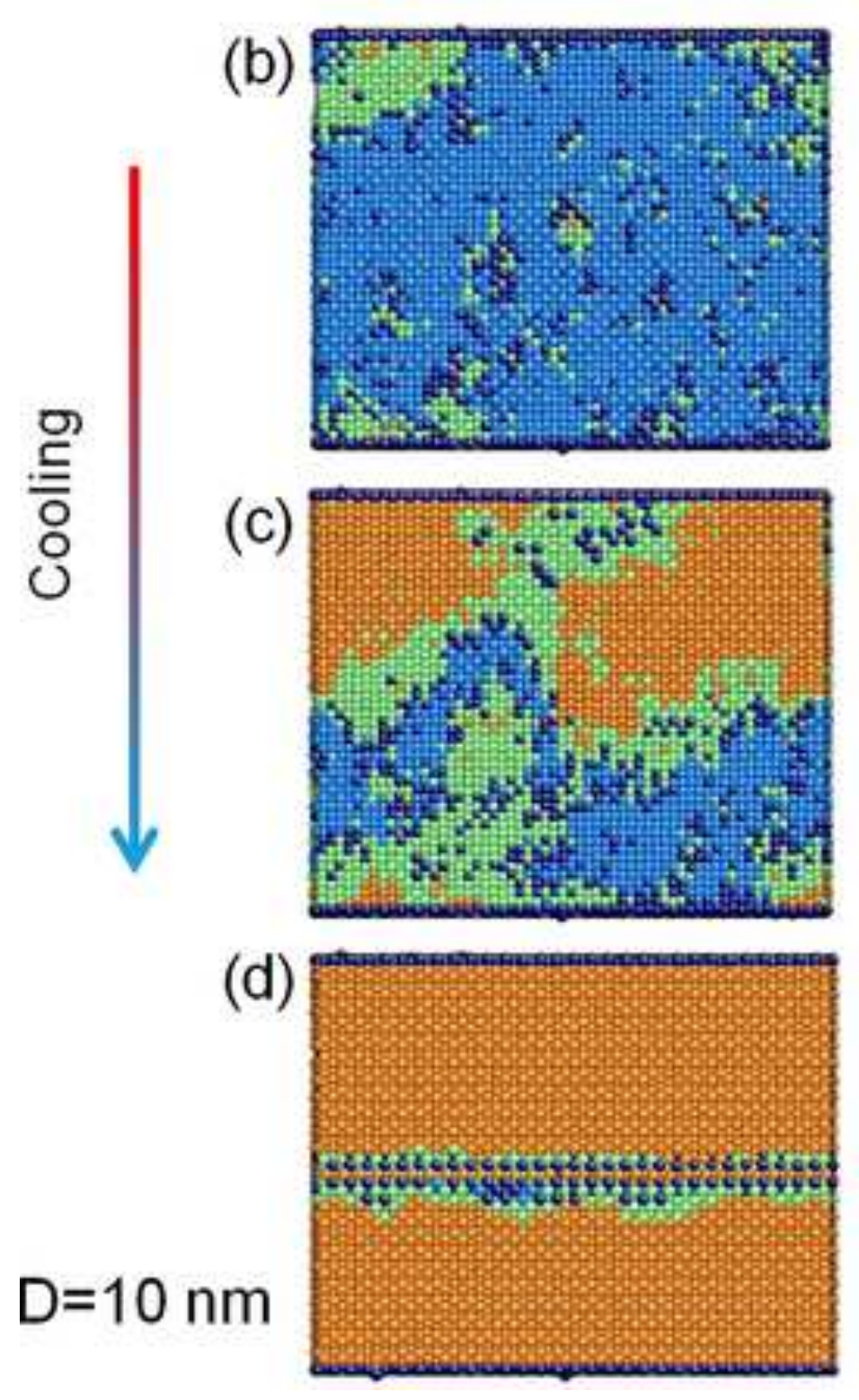

흠

$D=10 \mathrm{~nm}$ (d)
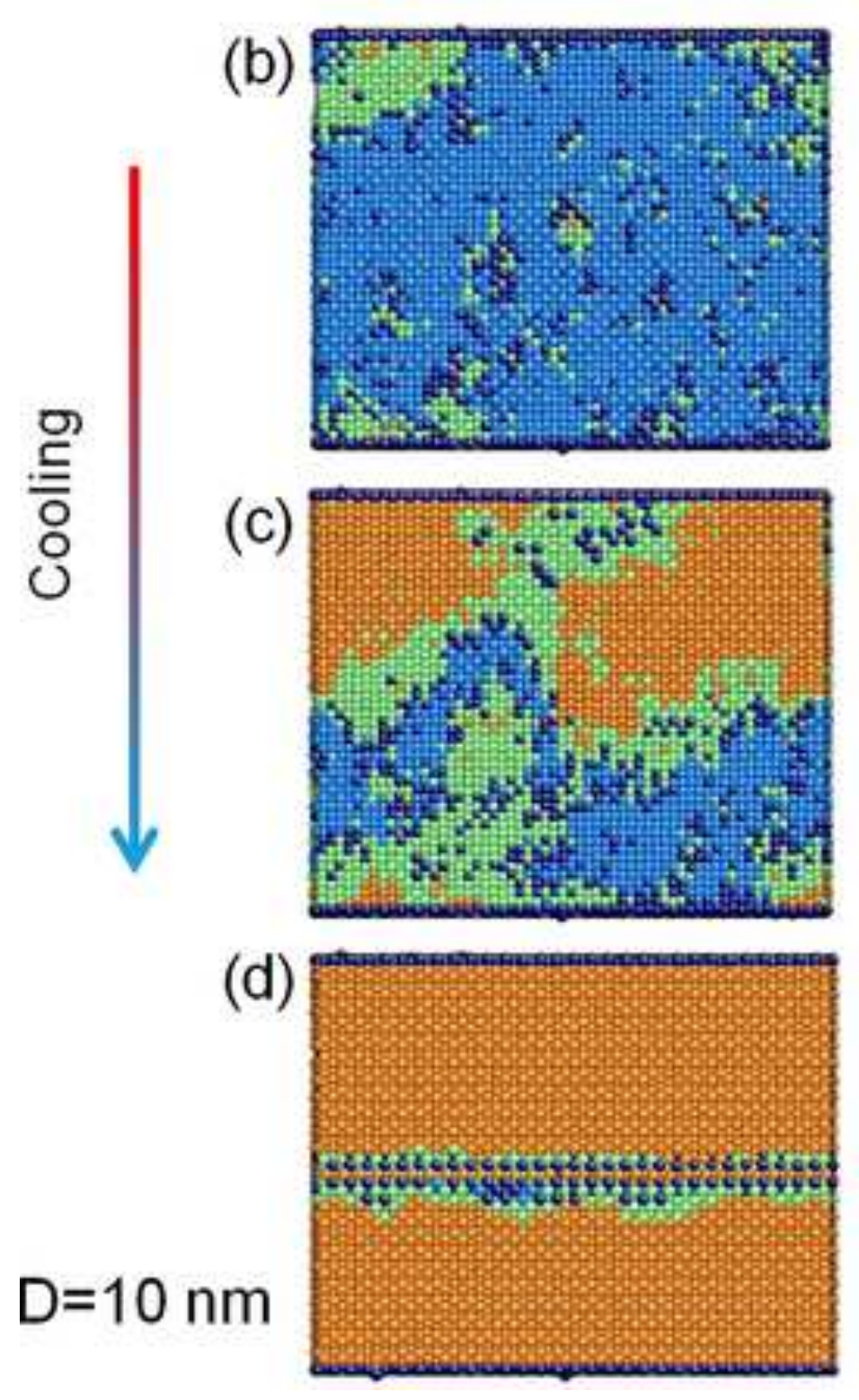

(h)

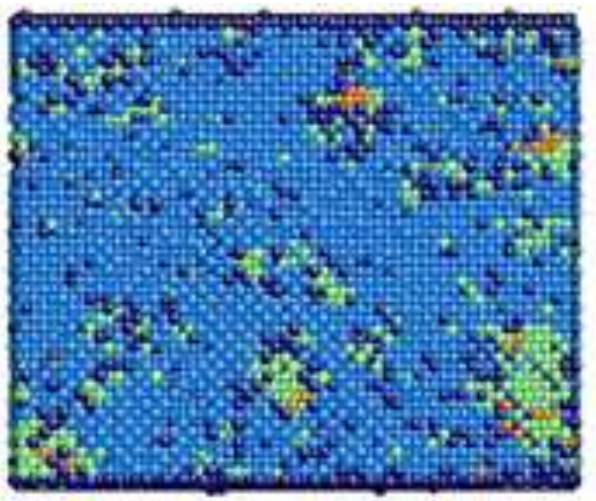

(g)

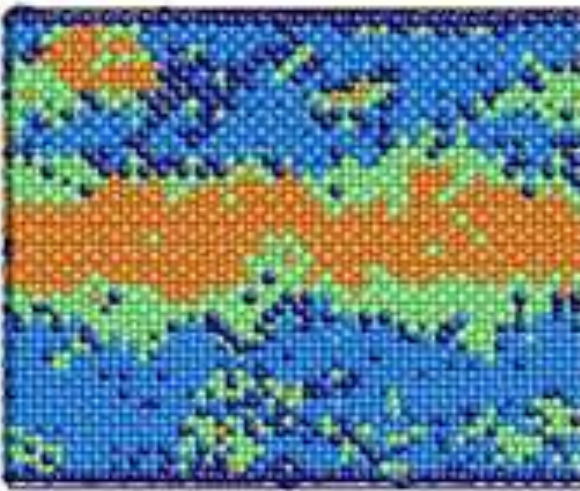

(f)
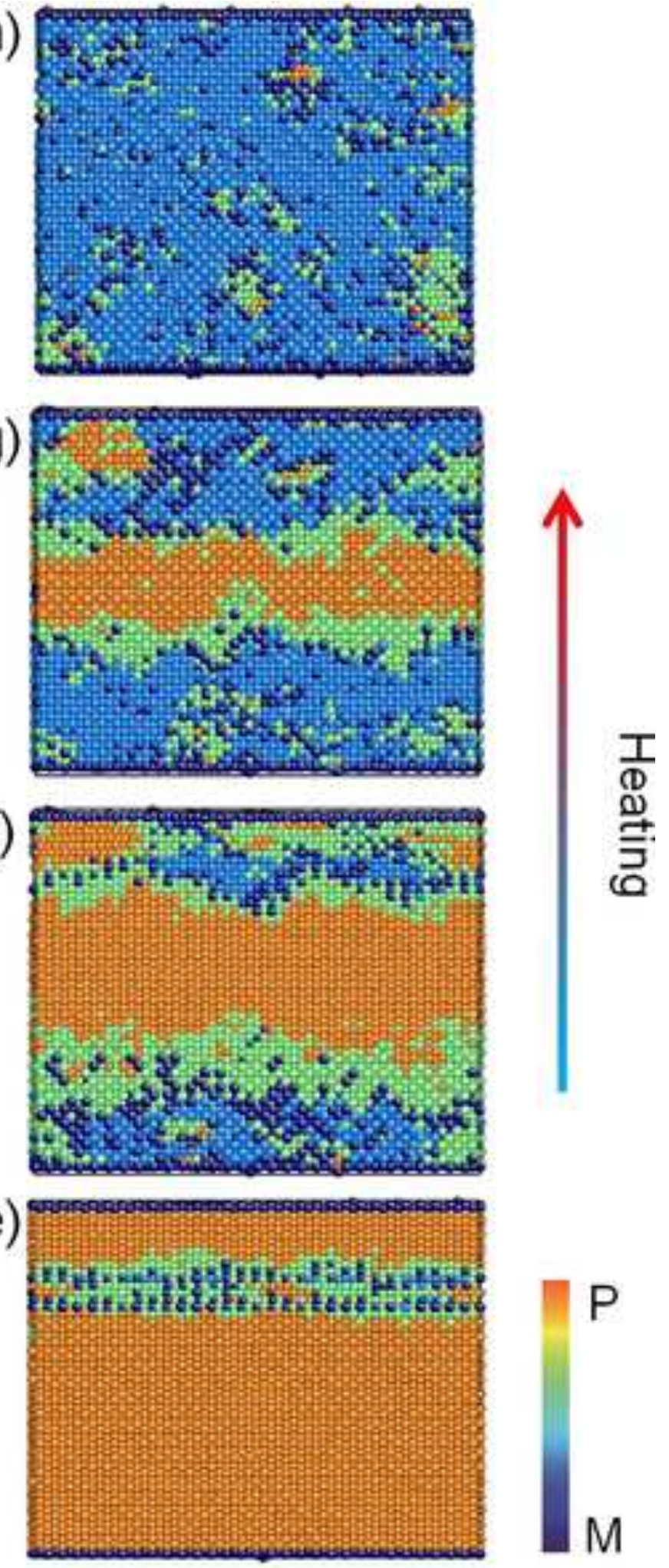

1$$
\text { . }
$$

(e) 

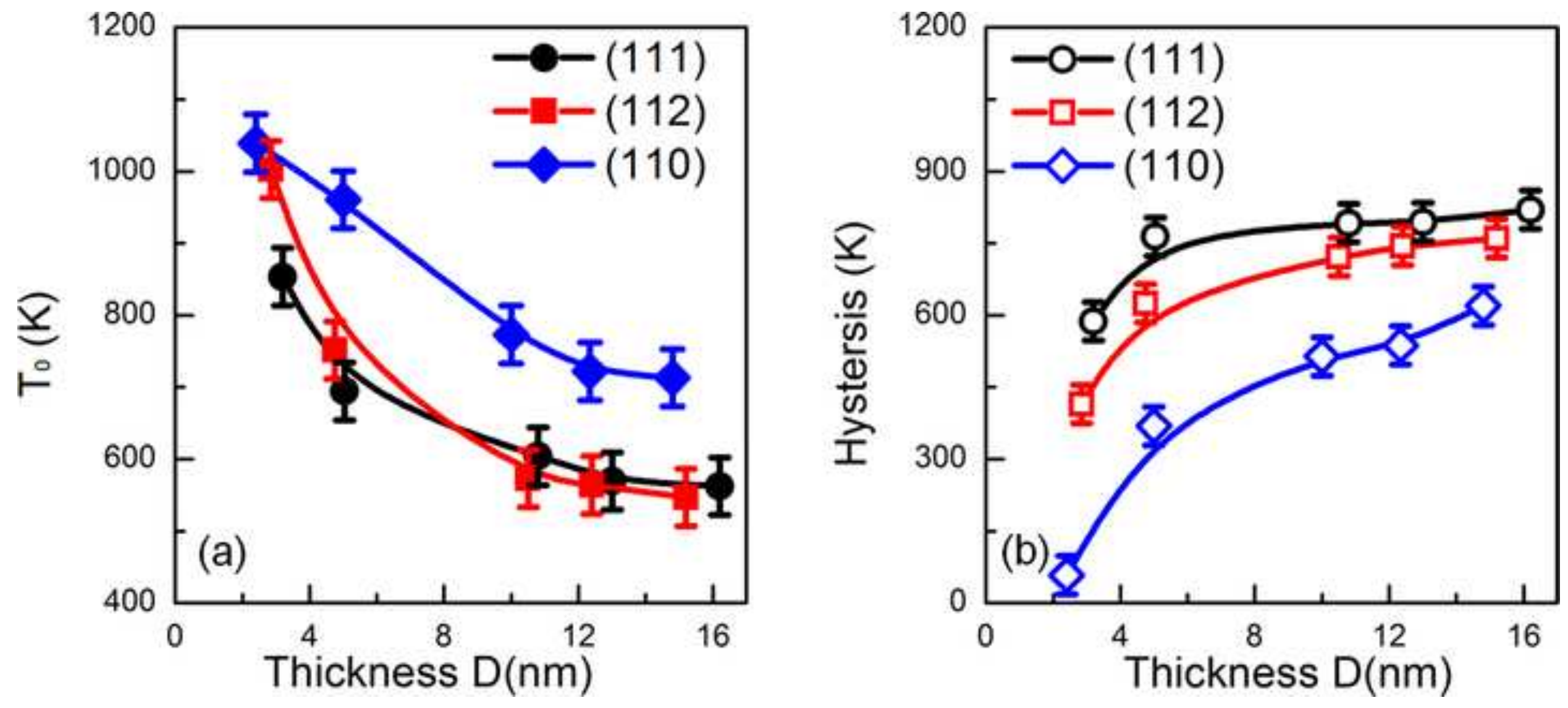
(110) Monolayer

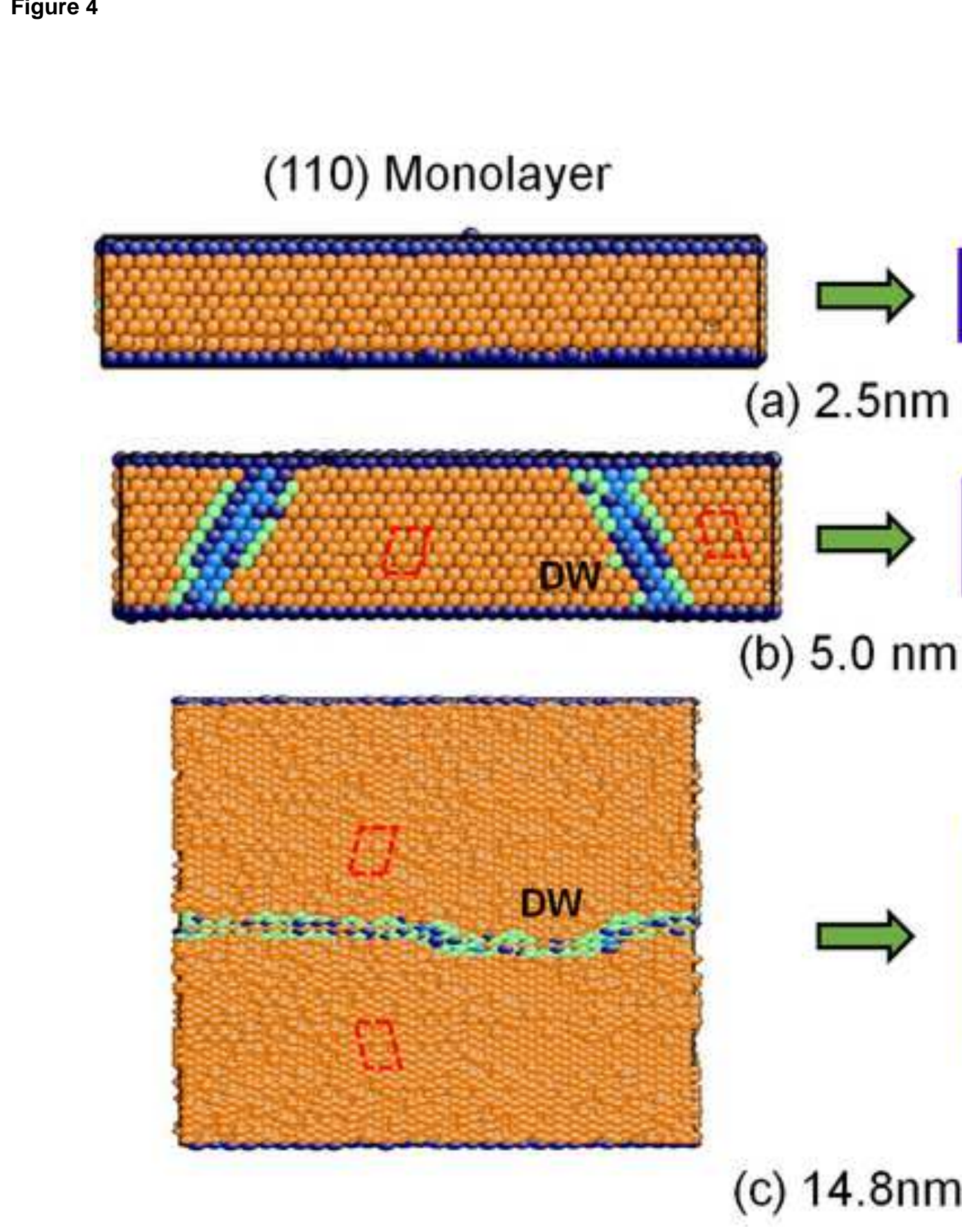

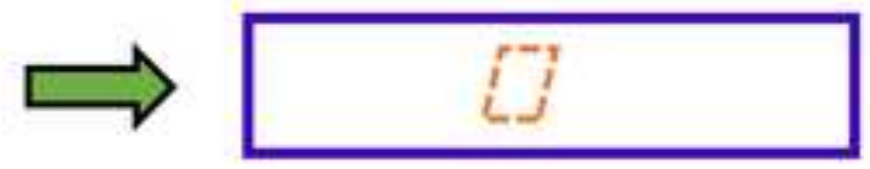

(a) $2.5 \mathrm{~nm}$

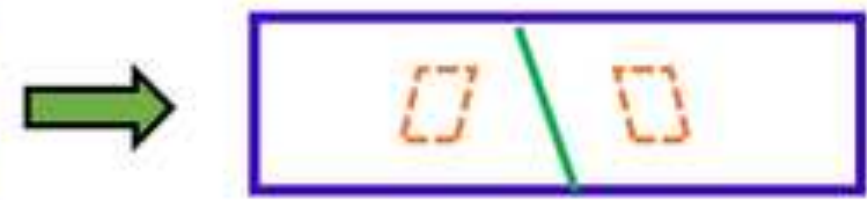

(b) $5.0 \mathrm{~nm}$

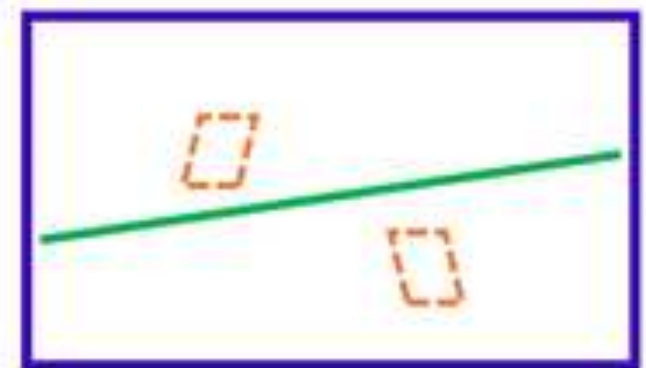

Out-of-plane
In-plane

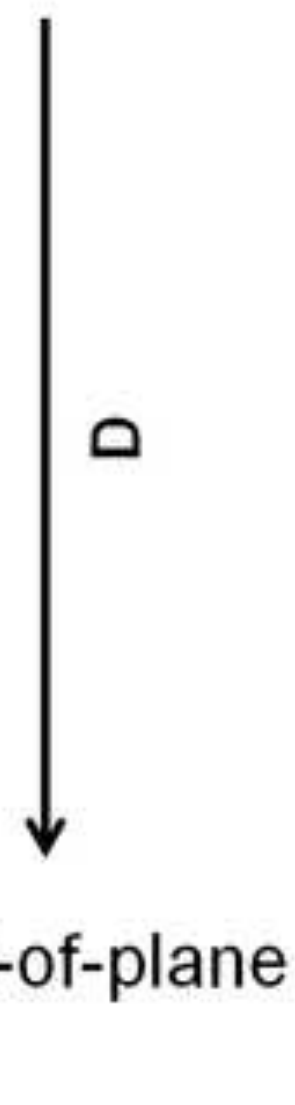

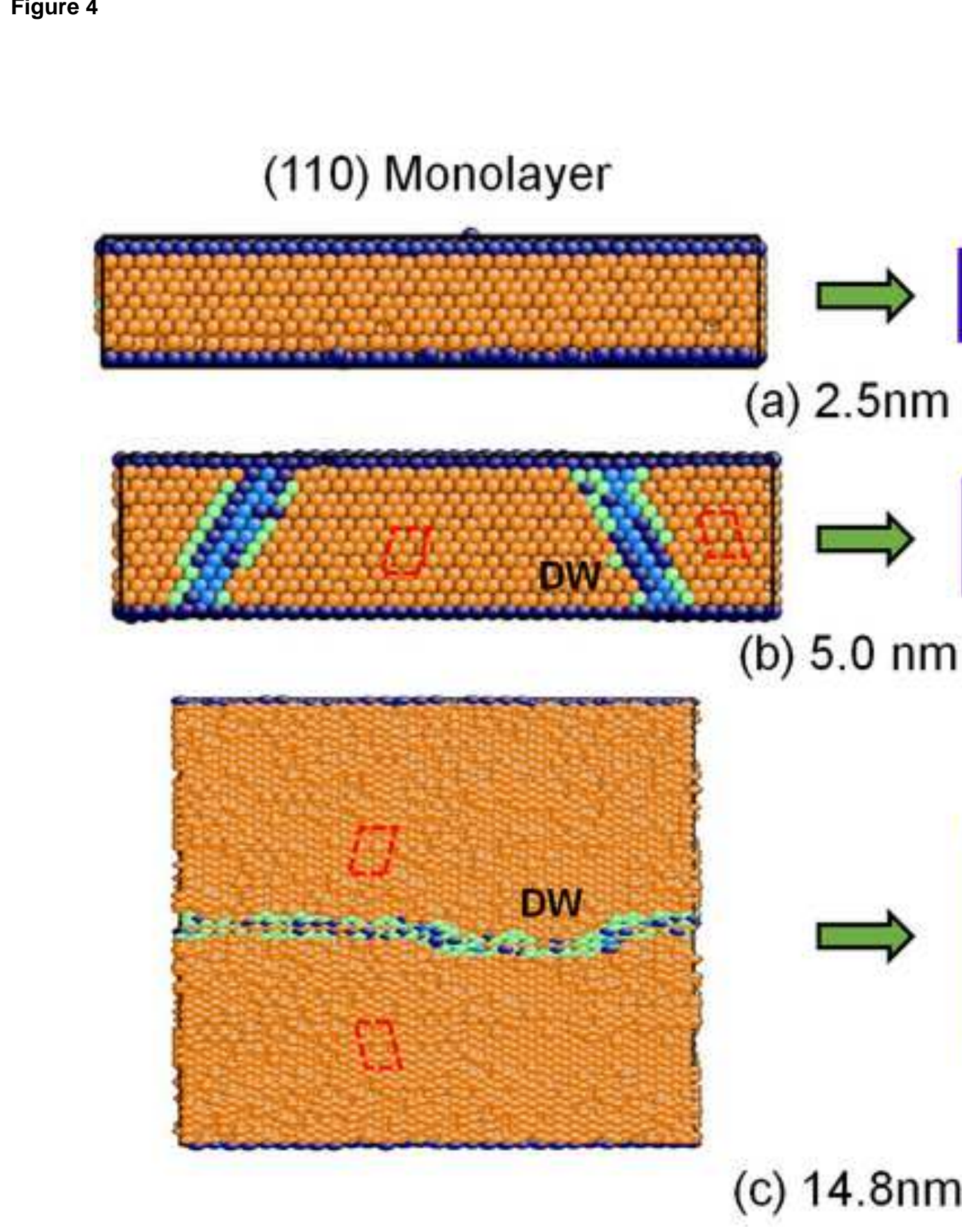
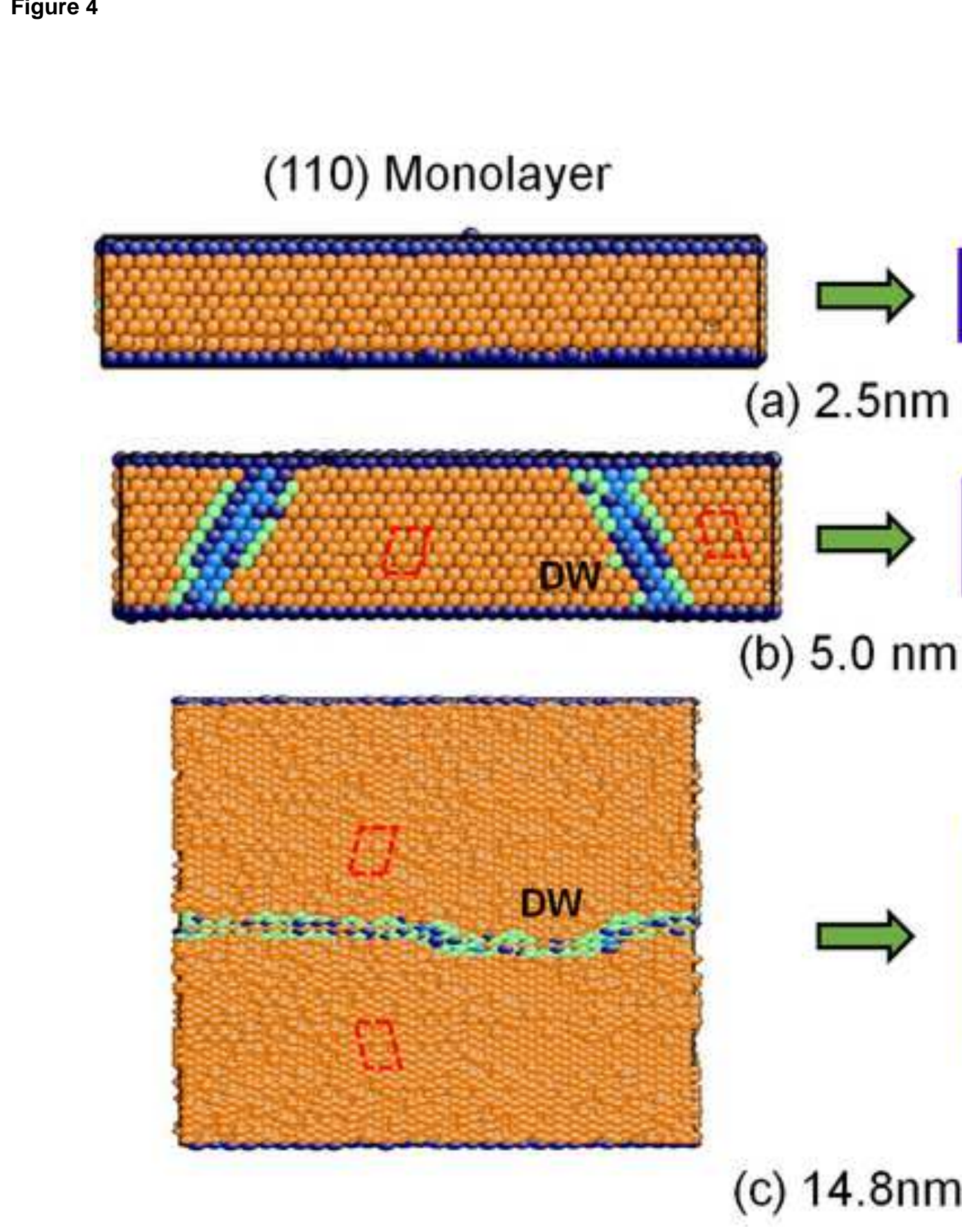
(c) $14.8 \mathrm{~nm}$ 

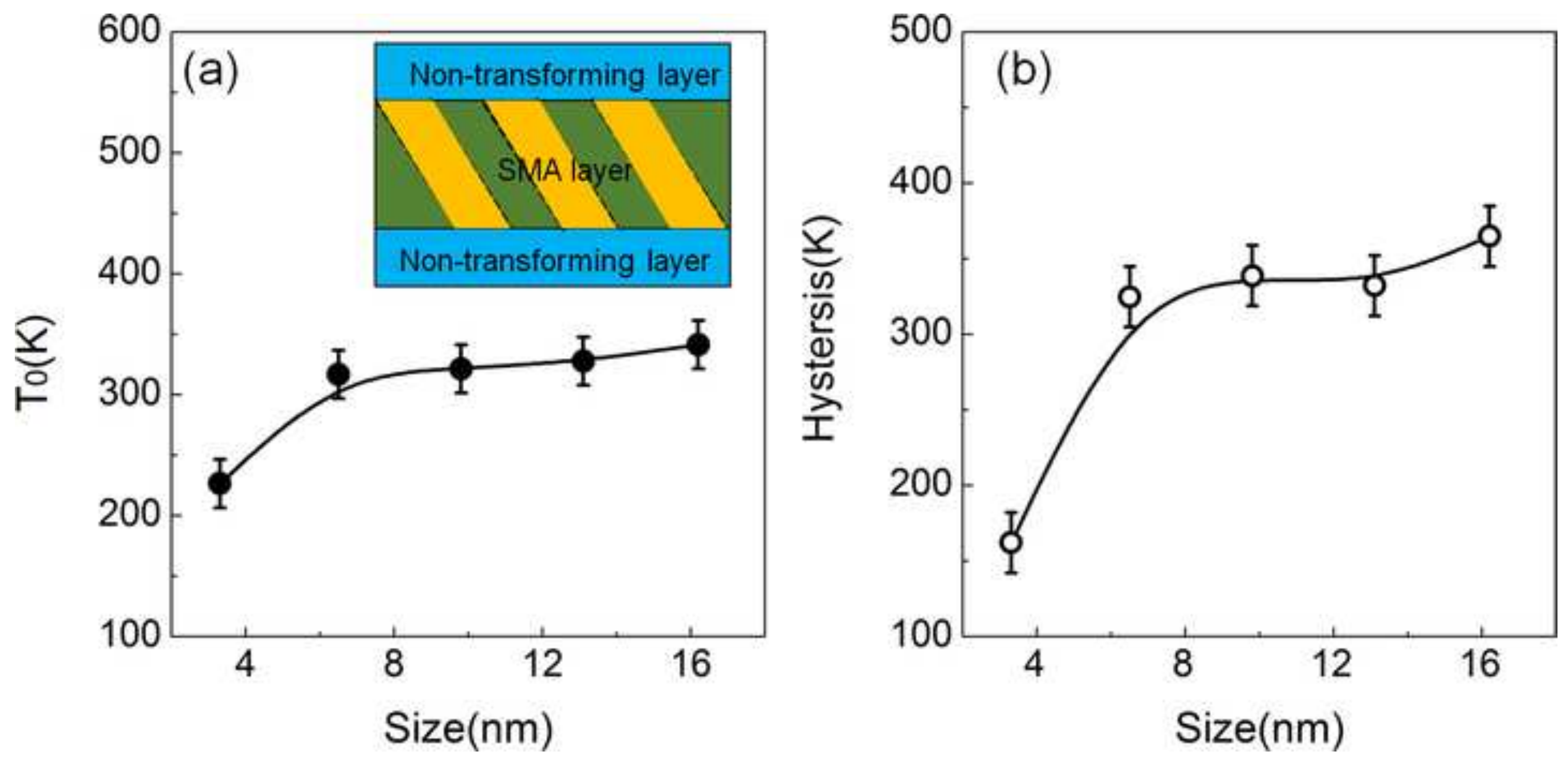


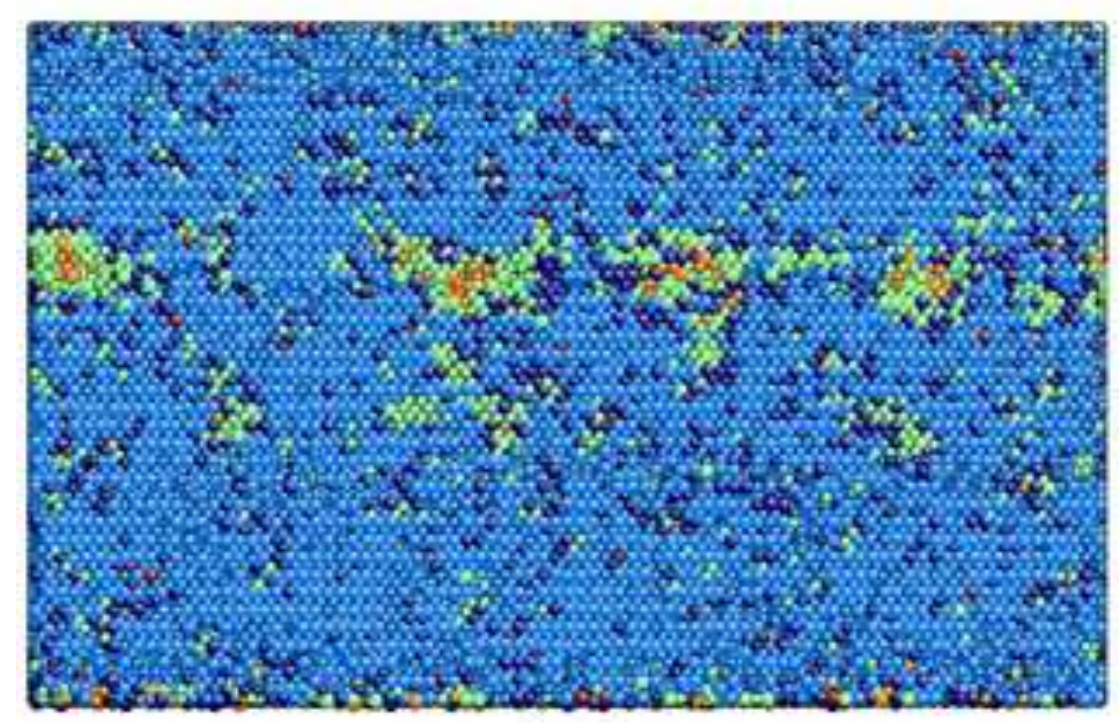

(a)

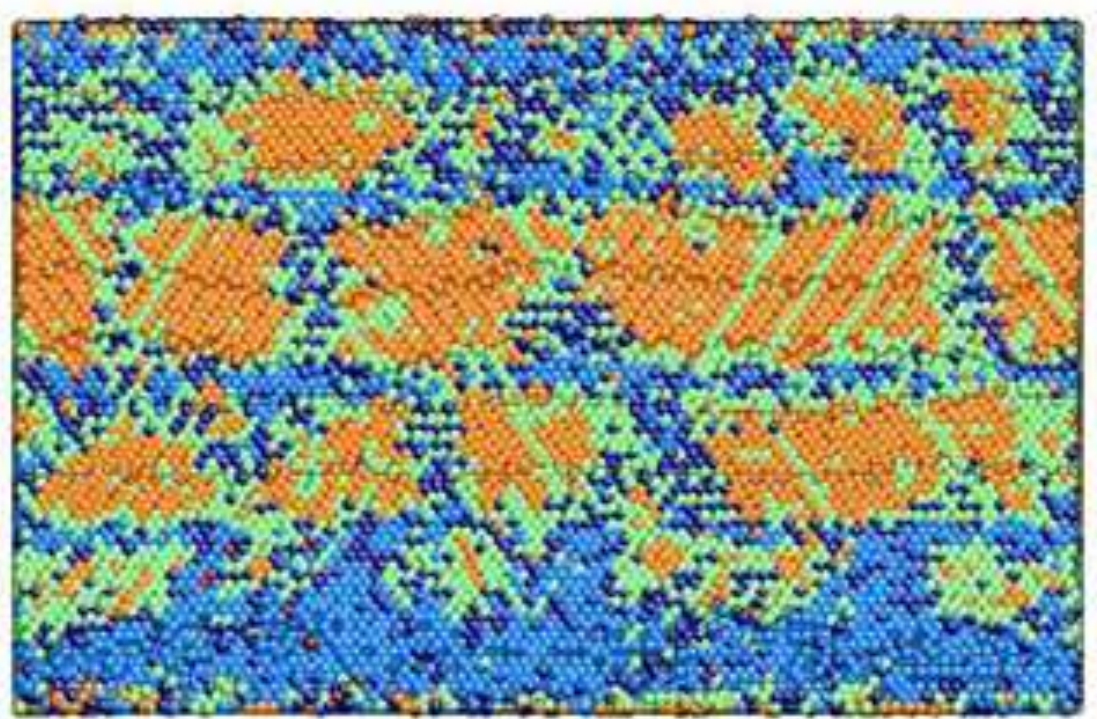

(c)

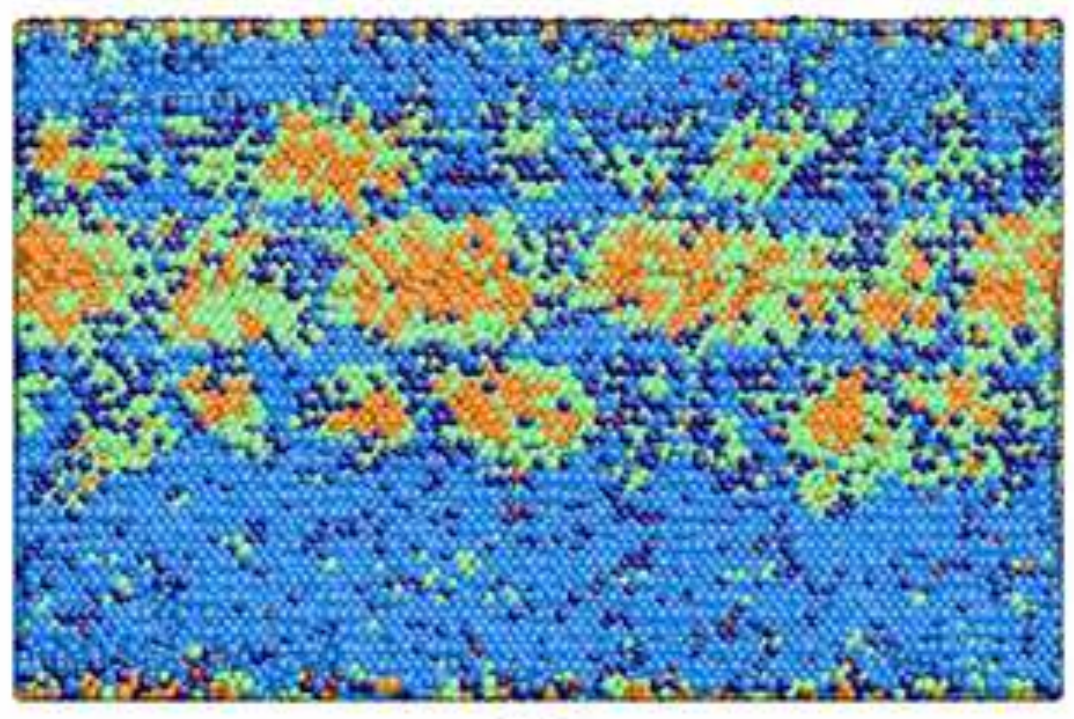

(b)

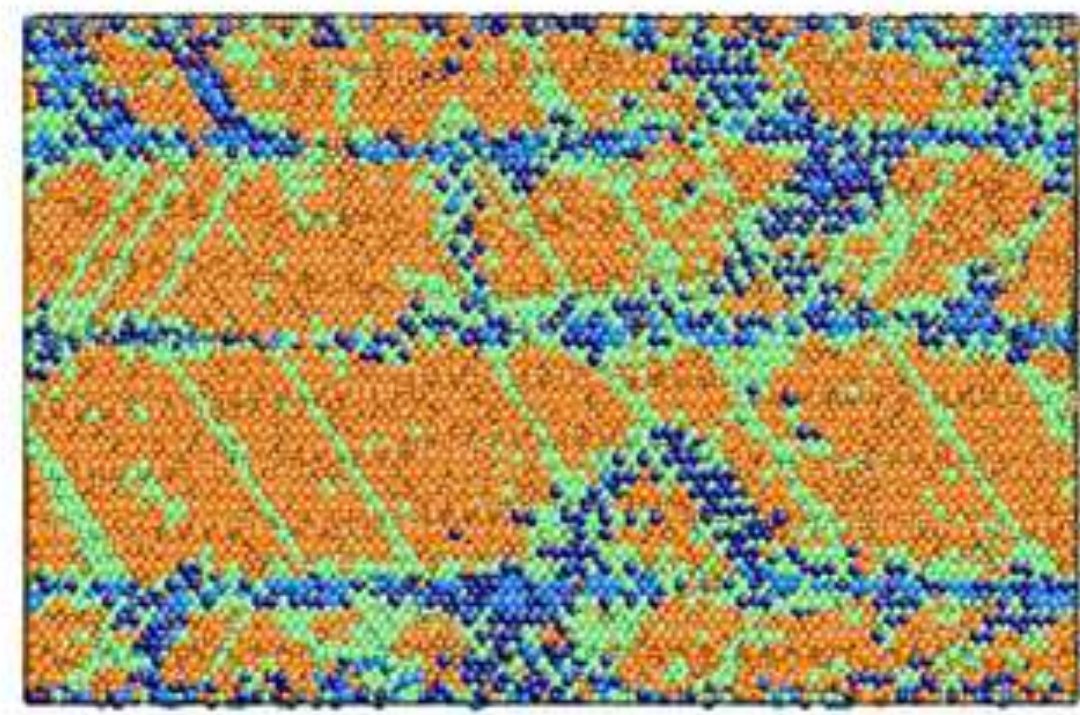

(d) 

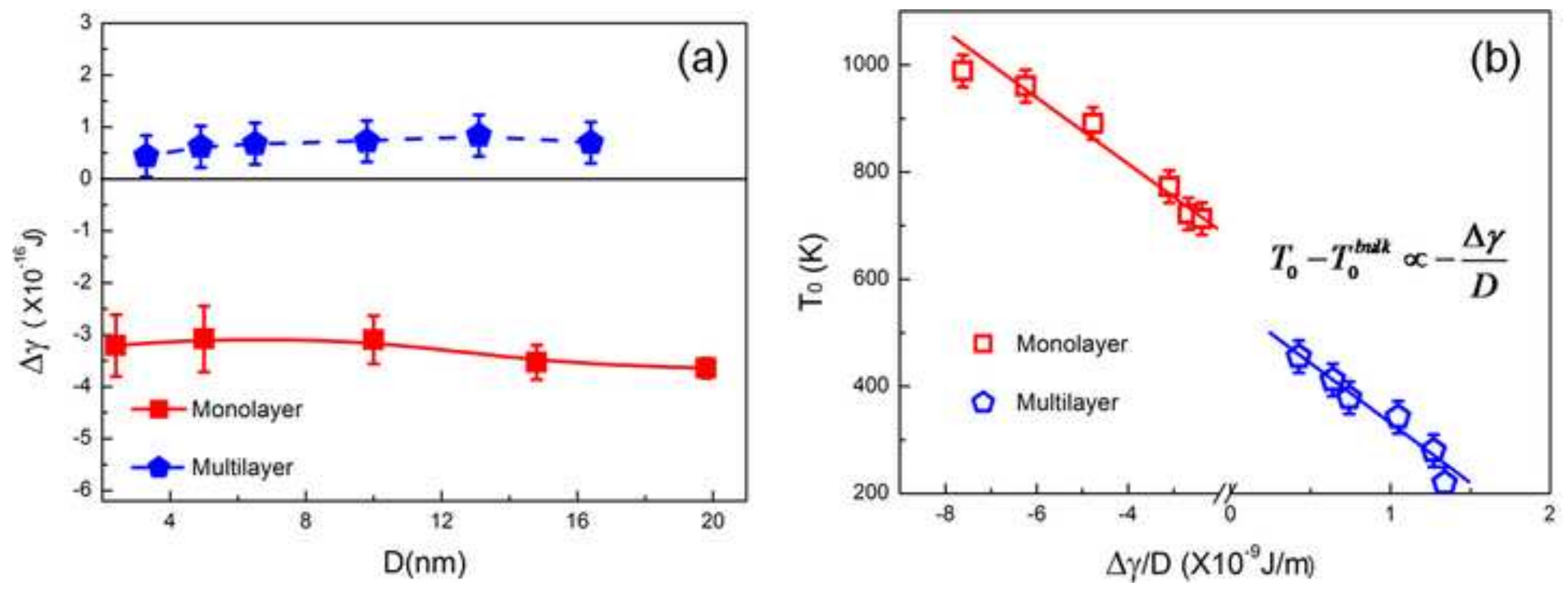

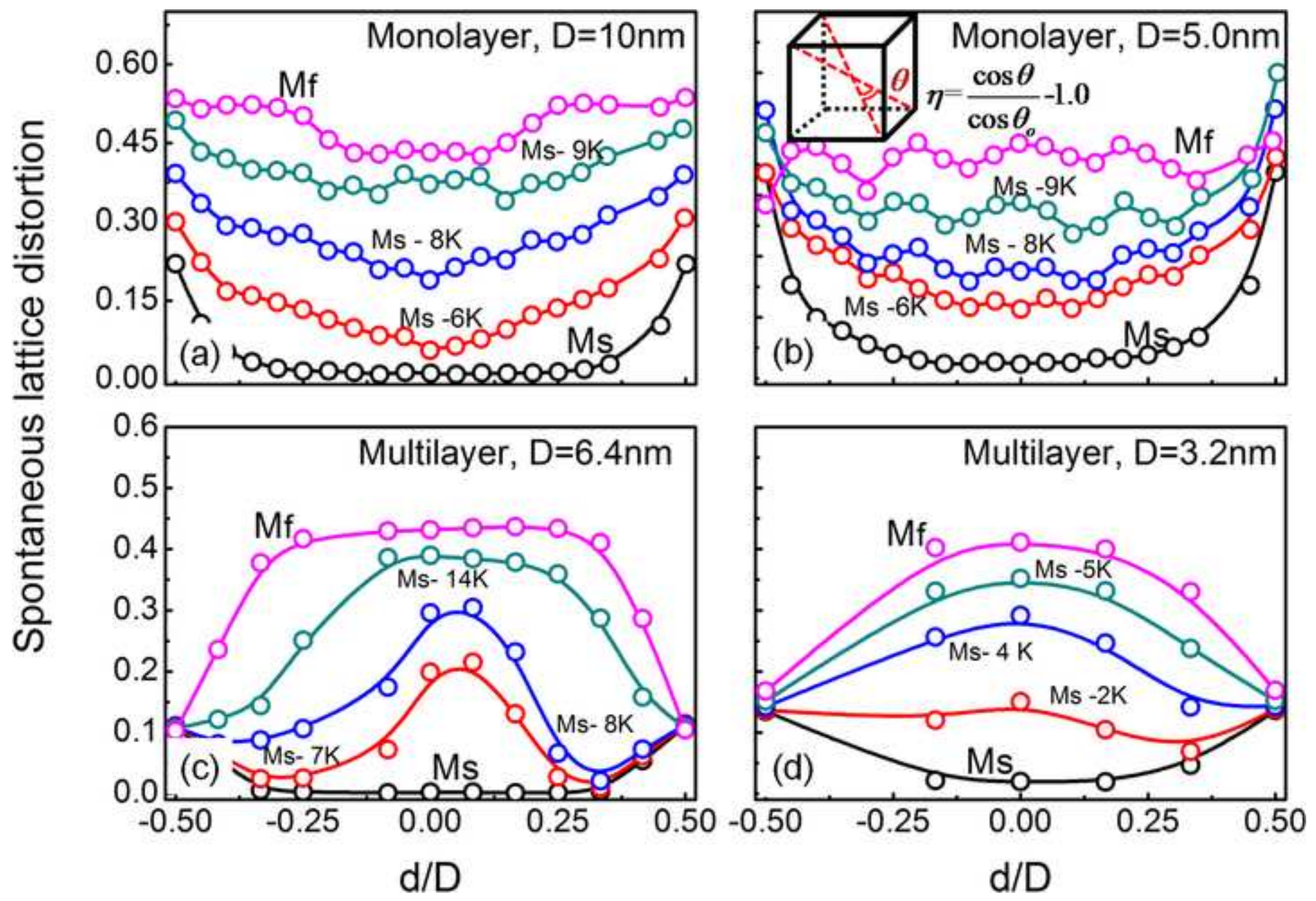
(a)

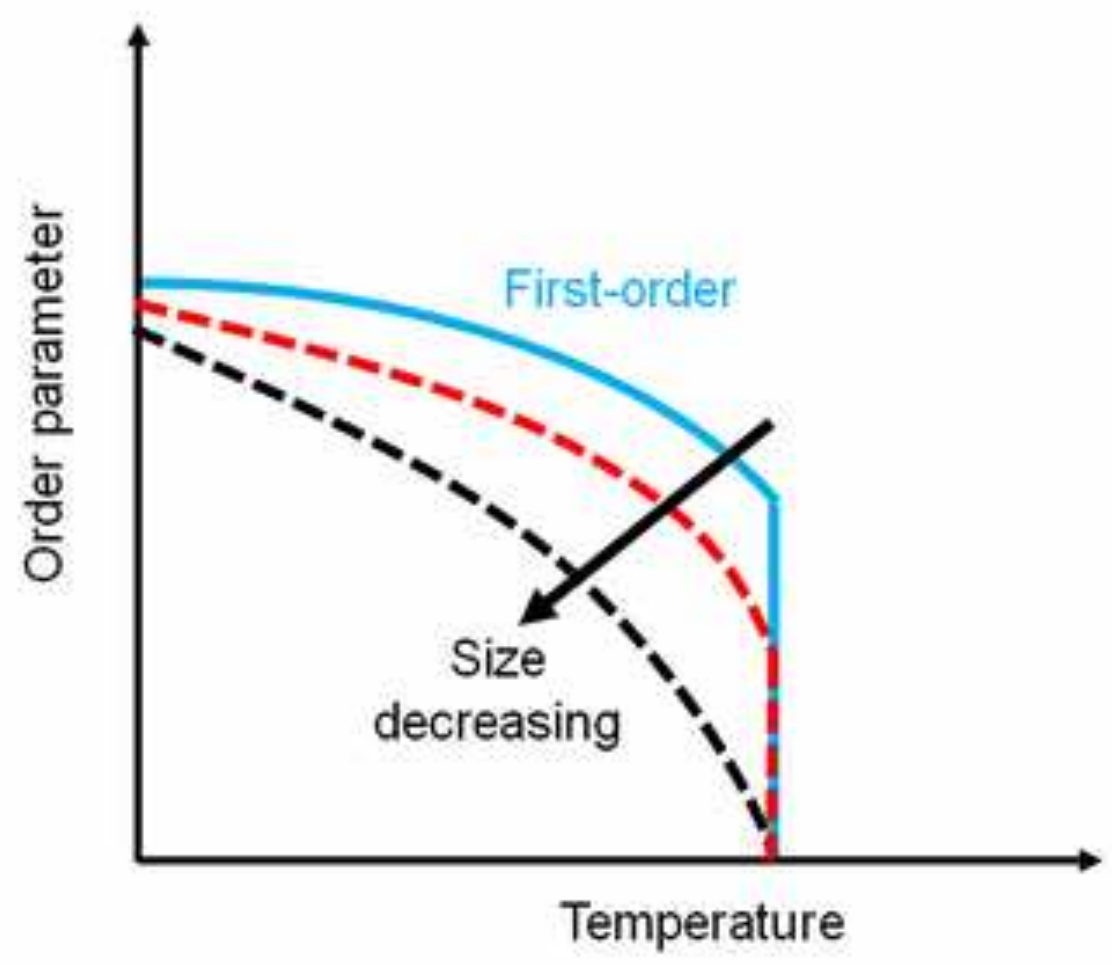

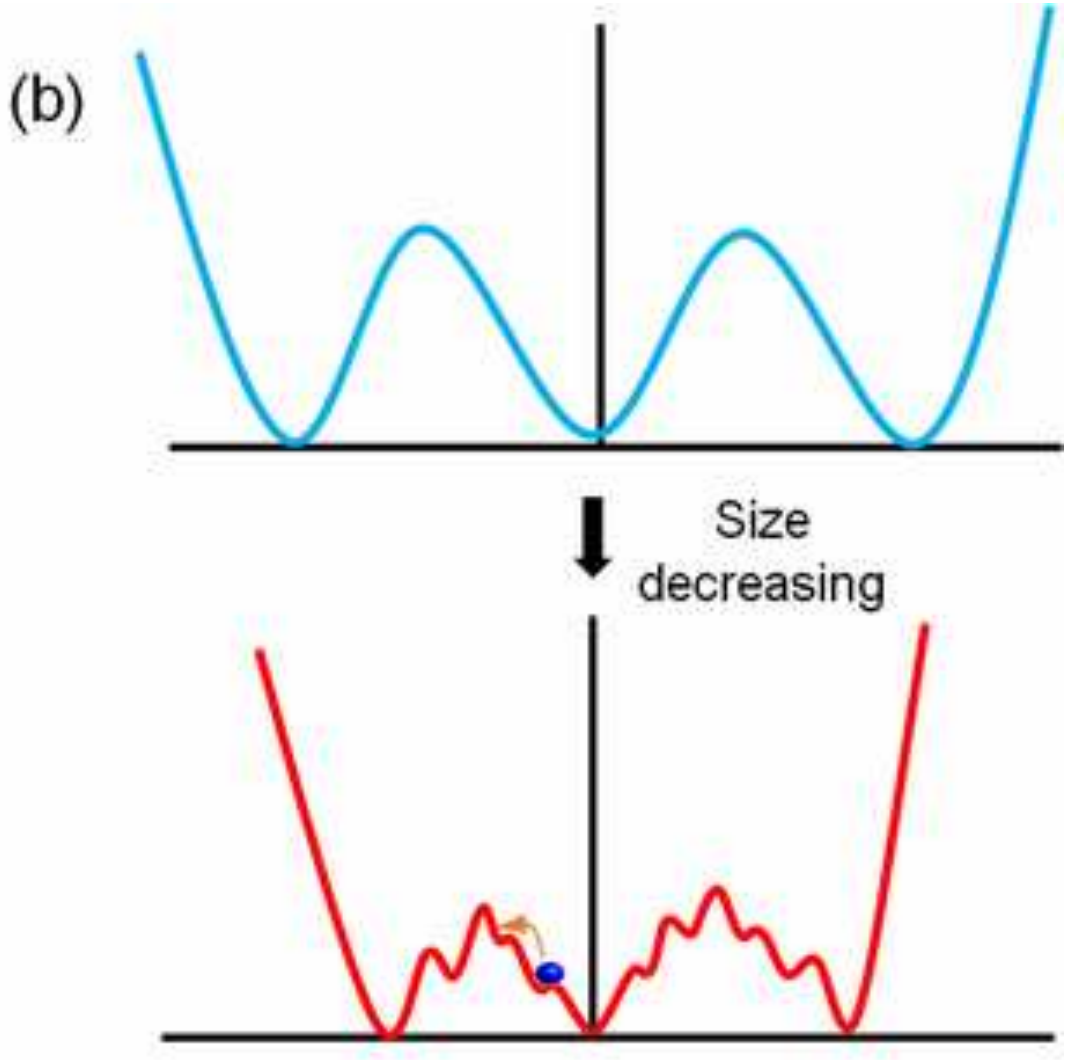



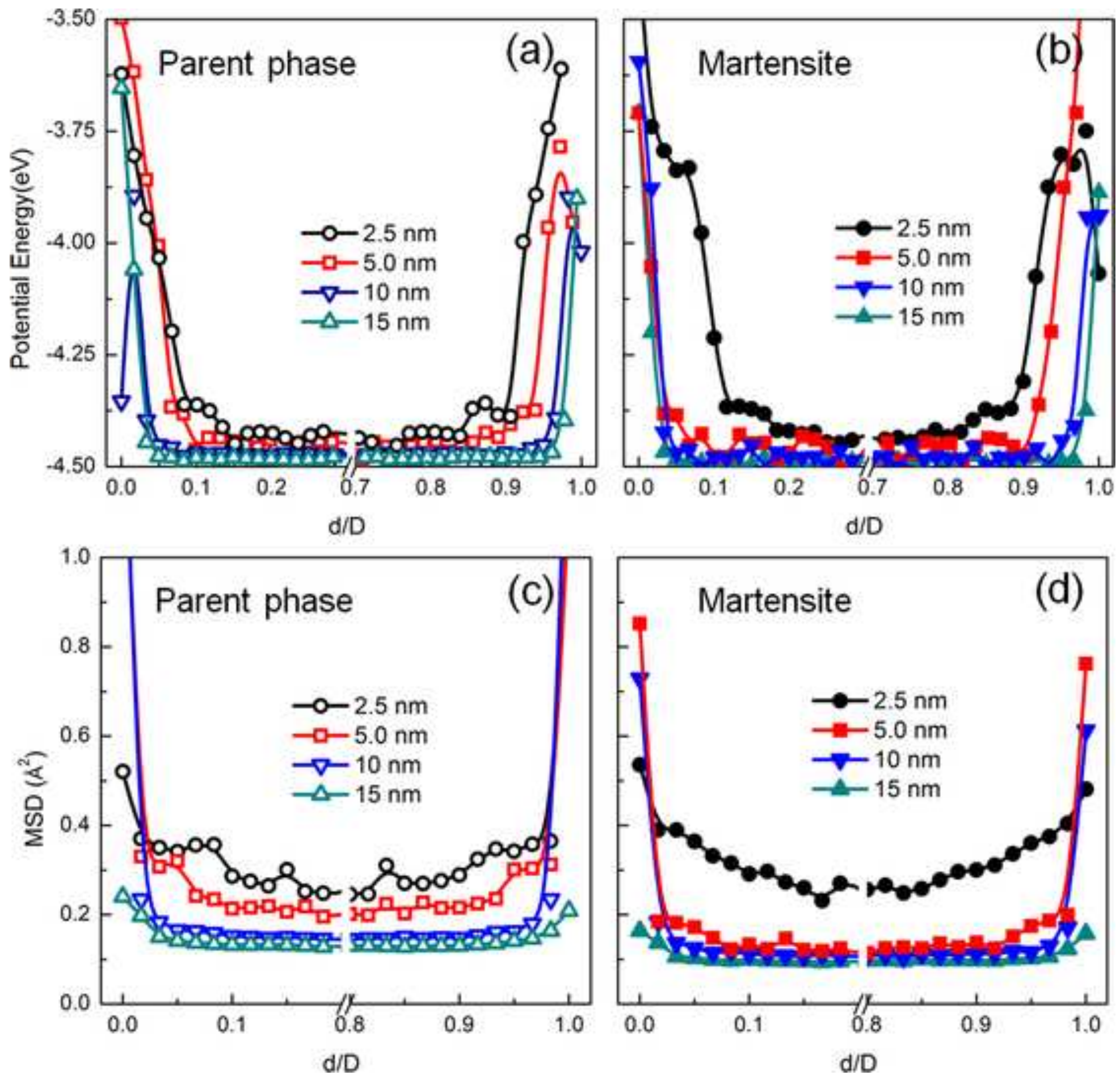


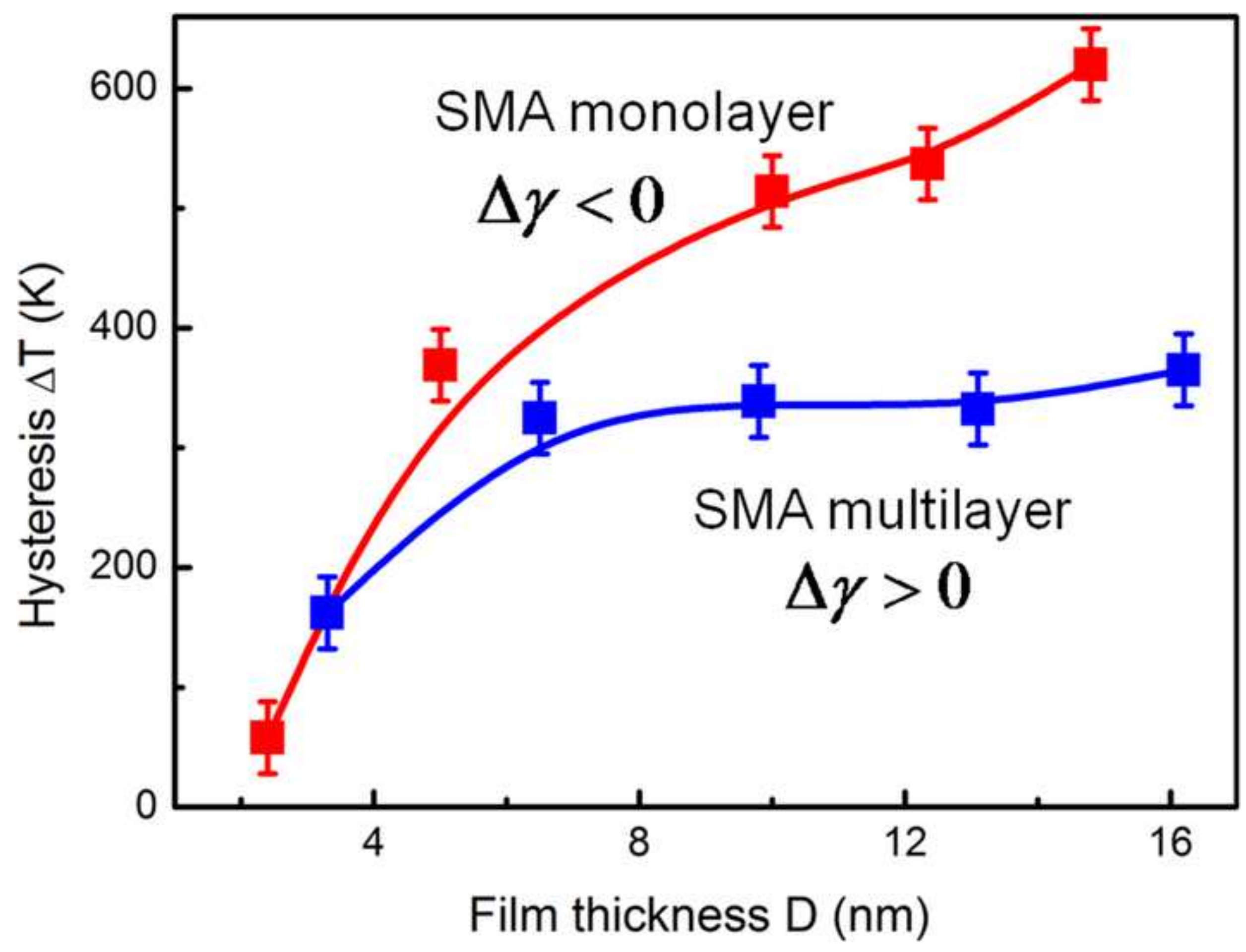

